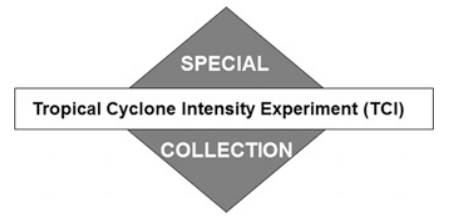

\title{
The Outflow-Rainband Relationship Induced by Environmental Flow around Tropical Cyclones
}

\author{
Yi DAI, Sharanya J. MAJUmDar, AND DAVID S. NOLAN \\ Rosenstiel School of Marine and Atmospheric Science, University of Miami, Miami, Florida
}

(Manuscript received 23 July 2018, in final form 3 February 2019)

\begin{abstract}
This study investigates the role of the asymmetric interaction between the tropical cyclone (TC) and the environmental flow in governing the TC inner-core asymmetric structure. Motivated by the limitations of bulk measures of vertical wind shear in representing the complete environmental flow, the TC outflow is used as a focus for the asymmetric interaction. By analyzing an idealized numerical simulation, it is demonstrated that parcels can go directly from the asymmetric rainband to the upper-level outflow. The relatively large vertical mass flux in the rainband region also suggests that the asymmetric rainband is an important source of the outflow. In a simulation that suppresses convection by reducing the water vapor within the rainband region, the upper-level outflow is weakened, further supporting the hypothesis that the rainband and outflow are directly connected. Finally, it is demonstrated that the asymmetric outflow and the outer rainband are coupled through the descending inflow below the outflow. Some of the main characteristics of the outflow-rainband relationship are also supported by a real-case numerical simulation of Hurricane Bill (2009). The relationship is potentially useful for understanding and predicting the evolution of the TC inner-core structure during the interaction with the large-scale environmental flow.
\end{abstract}

\section{Introduction}

The tropical cyclone (TC)-environmental flow interaction (TCEFI) plays an important role in TC structure and intensity change. Although some indices and methods exist to represent the TCEFI, they have mainly been developed in an axisymmetric framework. However, the azimuthally asymmetric interaction also needs to be considered, because the environmental flow is often highly asymmetric relative to the TC, thus creating an asymmetric forcing on the TC. Environmental features such as upper-level troughs and westerly jets can interact strongly with the TC. Whether the environmental flow is beneficial or detrimental to TC development depends mainly on the relative strength of the environmental features and the TC, and the distance between them (Hanley et al. 2001; Peirano et al. 2016). In those papers, the TC development means the axisymmetric response of the TC to the environmental flow. The eddy flux momentum convergence (EFC) is a good indicator of TCEFI, with the intensification of the TC associated with positive EFC (Pfeffer and Challa 1981;

\footnotetext{
Corresponding author: Yi Dai, ydai@rsmas.miami.edu
}

Molinari and Vollaro 1989). The EFC, together with the balanced Sawyer-Eliassen equation (Eliassen 1951), can be used to describe the eddy-induced radial and vertical components of the secondary circulation (Molinari and Vollaro 1990; Ditchek et al. 2017). Although the EFC combined with the Sawyer-Eliassen equation describes the eddy influence of the TCEFI, they are both built under the azimuthal-mean framework, meaning that they cannot explain the asymmetric structure caused by the TCEFI. Another method, the "asymmetric balanced" (AB) theory (Shapiro and Montgomery 1993), was also developed to describe the impacts of the asymmetric environmental forcing on TC development. However, it has primarily been used to study the axisymmetric response of TCs, such as the azimuthal-mean tangential wind tendency.

The influence of vertical wind shear (VWS) has been widely used to explain the asymmetric convective structure caused by the TCEFI. Here, the deep-layer VWS around a TC is defined by the 200-850-hPa wind vector difference averaged in a $200-800-\mathrm{km}$ annulus relative to the TC center (we refer to this as $\mathrm{VWS}_{200-800}$ ). One of the effects of the VWS is that the convection is located in the downshear-left quadrant of the TC, which has been found both in observational studies 

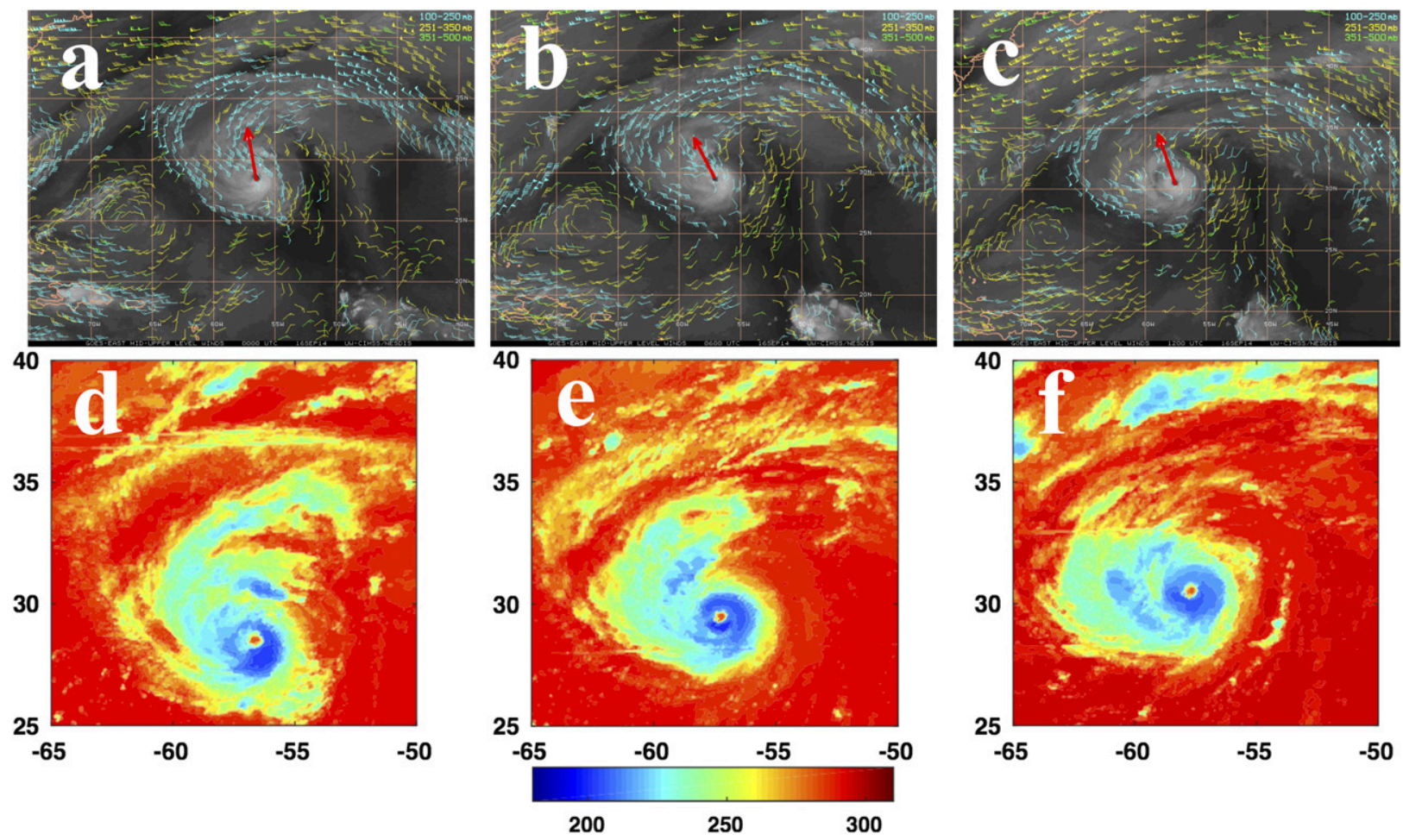

FIG. 1. (a)-(c) Upper-level atmospheric motion vectors (AMVs) superposed on the GOES-East water vapor images of Hurricane Edouard at 0000, 0600, and 1200 UTC 16 Sep 2014. The red arrow represents the calculated vertical wind shear $\left(\sim 8 \mathrm{~m} \mathrm{~s}^{-1}\right)$ using ERA-Interim data. (d)-(f) As in (a)-(c), but for the corresponding infrared brightness temperature (K).

(e.g., Corbosiero and Molinari 2003; Chen et al. 2006) and numerical experiments (e.g., Rogers et al. 2003; Riemer et al. 2010). Therefore, in simple terms, the VWS is able to relate the larger-scale environmental flow with the asymmetric convection outside the eyewall (in the rainband).

It initially appears as though the VWS can predict the asymmetric TCEFI process sufficiently well. However, the fact that VWS is merely the wind difference between two levels may lead to inaccurate representations of the full environmental flow, as identified in recent studies. For example, in numerical simulations (e.g., Nolan 2011; Finocchio et al. 2016) in which the VWS is kept the same but the vertical wind profile is varied, substantially different TC structure and intensity evolutions are produced. Furthermore, recent studies have focused on the importance of considering VWS that varies smoothly in time (Onderlinde and Nolan 2017; Finocchio and Majumdar 2017). Therefore, the use of a single value of VWS, without additional consideration of its time evolution, may not be sufficient to demonstrate the full range of TCEFI, and we suggest that the detailed structure of the environmental flow as well as its evolution is also important for TC intensity and structure.
Moreover, the direction of the $\mathrm{VWS}_{200-800}$ does not always represent that of the environmental flow, especially when a TC is present. For example, when Hurricane Edouard (2014) interacted with a southwesterly jet before reaching its maximum intensity (Fig. 1), the direction of $\mathrm{VWS}_{200-800}$ (red arrow in the top panels of Fig. 1) was to the north-northwest, nearly perpendicular to the jet direction. In addition to the inconsistency between the direction of the VWS and that of the real environmental flow, the "downshear left" argument of the VWS also does not always hold. At 1200 UTC 16 September 2014, most of the convection outside the eyewall of Edouard was located to the upshear left (Fig. 1f), consistent with the findings of Rogers et al. (2016) for the same TC. Looking at the time evolution of Edouard, the convection outside the eyewall turned counterclockwise with time (Figs. 1d-f), while the VWS vector (Figs. 1a-c) turned counterclockwise first, and then clockwise with time. Interestingly, the outflow of Edouard, which can be visualized by the anticyclonic blue wind barbs (atmospheric motion vectors, to be described in section 2) surrounding the TC center, evolved in a similar way as the convection: that the southern edge of the outflow (Figs. 1a-c) became flatter with time, meaning that it also turned counterclockwise. 

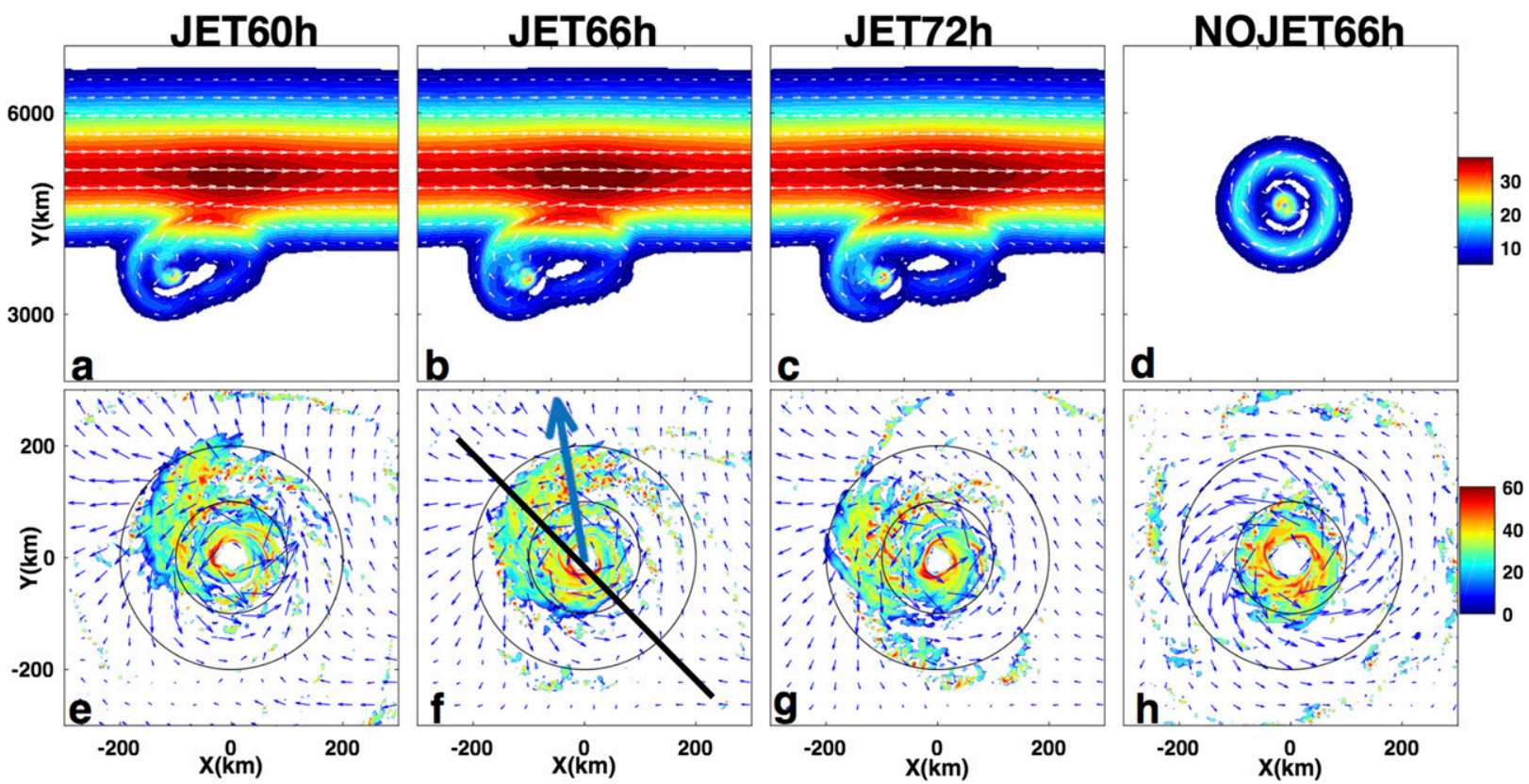

FIG. 2. Overview of the JET simulation from DMN17. (a)-(c) Horizontal wind speed (shaded) and horizontal wind vectors (white arrows) at $p=200 \mathrm{hPa}$ at model times of 60,66 , and $72 \mathrm{~h}$ in the JET simulation, with the domain size of $5000 \mathrm{~km} \times 5000 \mathrm{~km}$. (d) As in (b), but for the NOJET simulation. (e)-(h) The corresponding TC inner-core $(600 \mathrm{~km} \times 600 \mathrm{~km})$ reflectivity (shaded) at $z=2 \mathrm{~km}$ and horizontal wind vectors (blue arrows) at $z=12 \mathrm{~km}$. The large blue arrow in (f) indicates the vertical wind shear (value is $5.7 \mathrm{~m} \mathrm{~s}{ }^{-1}$ ). The two circles represent $r=100$ and $200 \mathrm{~km}$.

This inconsistency between the VWS and convection not only occurred in Hurricane Edouard (2014). In a recent idealized simulation of the TC interacting with a westerly jet (Dai et al. 2017, hereafter DMN17), the upper-level environmental flow is to the east, but the VWS vector is directed nearly to the north (blue arrow in Fig. 2f). Why is the $\mathrm{VWS}_{200-800}$ not consistent with the large-scale environmental flow? It turns out that the defined 200-800-km annulus was too small to cover the major environmental flow for this simulated TC. Instead, the calculated VWS only reflects the TC upperlevel outflow, and is just a manifestation of the outflow channel (at least in the case of Edouard and in this case for DMN17), which is a consequence of the TCEFI.

Motivated by the limitations of the VWS in fully representing the complex environmental flow in both Hurricane Edouard (2014) and the simulations in DMN17, we seek a more thorough physical explanation of the TCEFI and the TC asymmetric inner-core structure. In particular, we ask whether the TC outflow plays an important role in the asymmetric structural evolution, and if so, how. As the upper part of the TC secondary circulation, the outflow always organizes as a concentrated channel or several channels (Chen and Gray 1985; Ooyama 1987). In the upper-level outflow region, the inertial stability is very low (or even slightly negative) because of the TC anticyclonic wind. As a result, the outflow can travel thousands of kilometers horizontally and be easily shaped by environmental flows. In other words, the outflow can be thought as a passive indicator of the environmental flow. For example, when an upperlevel trough or jet is located to the north of a TC, a northward outflow channel always appears. Does this outflow have any relationship with the TC inner core structure? Another question about the outflow we would like to ask is the following: Does the source of the outflow always come from the eyewall? In the classical model of TC secondary circulation of Emanuel (1986), the upper-level outflow mainly came from the outwardsloping eyewall region [region II in Emanuel's (1986) Fig. 5]. But this conceptual model was built on the idea of a TC in a quiescent environment. Might a significant part of the outflow also come from outside the eyewall when there is a strong environmental interaction? In this paper, we test the hypothesis that the upper-level outflow channel is physically related to the asymmetric convection outside the eyewall around the TC inner core, and the environmental flow can influence the TC inner core via generation of the outflow channel.

In DMN17, the northward outflow channel is consistent with the asymmetric rainband area outside of the TC eyewall. In this study, we will use the simulation data from DMN17 as well as other data to show the connection between the asymmetric rainband and the TC outflow. 
The rest of the study is arranged as follows. Section 2 introduces the data and methodology, together with a brief overview of DMN17. In section 3, data from DMN17 as well as a test experiment will be used to test our hypothesis. Section 4 introduces the analysis of a realistic simulation of Hurricane Bill (2009). The sensitivity experiments and conclusions are provided in sections 5 and 6 , respectively.

\section{Data and methodology}

\section{a. Data sources and simulations}

To show the upper-level outflow channel and the synoptic-scale environmental flow, the upper-level atmospheric motion vectors (AMVs; Velden et al. 1997), together with water vapor satellite imagery, are used to study Hurricanes Edouard (2014) and Bill (2009). The AMV images are collected from the Cooperative Institute for Meteorological Satellite Studies (CIMSS) at the University of Wisconsin-Madison. This study also makes use of European Centre for Medium-Range Weather Forecasts (ECMWF) interim reanalysis (ERA-Interim; Dee et al. 2011) data to show upper-level flow features. The horizontal grid spacing of the ERA-Interim data is about $0.7^{\circ} \times 0.7^{\circ}$, which is sufficient to observe the outflow and environmental flow, but not enough to resolve the inner-core dynamics. As in the recent study by Fischer et al. (2017), the GridSat infrared (IR) brightness temperature, which has a horizontal resolution of about $0.07^{\circ}$ (Knapp et al. 2011), is adopted to visualize convection (or rainbands) around the TC inner core in real hurricanes.

For the majority of this study, we use data from Weather Research and Forecasting (WRF; version 3.4.1) Model simulations of an idealized TC interacting with an upper-level westerly jet on an $f$ plane (DMN17). The modeling framework contains three domains with horizontal grid spacing of 18,6 , and $2 \mathrm{~km}$, in domain sizes of $10800 \mathrm{~km} \times 7200 \mathrm{~km}, 1152 \mathrm{~km} \times 1152 \mathrm{~km}$, and $778 \mathrm{~km} \times 778 \mathrm{~km}$, therefore containing $600 \times 400,192 \times$ 192 , and $384 \times 384$ grid points, respectively. Vertically, 40 equally spaced levels are chosen in the WRF normalized hydrostatic pressure coordinate between the surface and approximately $z=20 \mathrm{~km}$. The zonal boundaries are periodic while free-slip boundary conditions are used on the northern and southern boundaries. Both radiation and cumulus parameterizations are not used in the model. The Yonsei University (YSU) scheme (Hong et al. 2006) is used for the planetary boundary layer; the WRF 5-class scheme (WSM5; Hong et al. 2004) is adopted for the microphysics parameterization. The initial TC is a modified Rankine vortex with a peak tangential wind speed of $20 \mathrm{~m} \mathrm{~s}^{-1}$ at the radius of maximum winds (RMW) of $90 \mathrm{~km}$. The westerly jet structure is adopted from Simmons and Hoskins
(1977), with the center of the jet around $z=12 \mathrm{~km}$. All the simulations are set on an $f$ plane with $f_{\text {cor }}=6.16 \times 10^{-5} \mathrm{~s}^{-1}$ (Coriolis parameter at $25^{\circ} \mathrm{N}$ ).

To investigate how air parcels in the outflow and rainband are connected, we conduct a trajectory analysis following the method of Draxler and Hess (1998). Using this method, the 4D database (both spatially and temporally) is interpolated to each trajectory by the second-order Runge-Kutta linear interpolation. To create accurate trajectories, we use the model output with 2-min temporal resolution over a period of $12 \mathrm{~h}$, and we make the interpolated time step $\Delta t=10 \mathrm{~s}$.

To further examine our hypothesis with a realistic TC, a simulation of Hurricane Bill (2009) is used to demonstrate the outflow-rainband relation. This simulation is closely modeled after the simulation of Hurricane Bill (2009) originally produced by Moon and Nolan (2015). This simulation used WRF, version 3.4.1, and simulated Bill from 1200 UTC 18 August to 0000 UTC 22 August 2009. The outer domain has 9-km grid spacing with $384 \times 336$ grid points, and there are two nested, vortex-following grids with 3 - and $1-\mathrm{km}$ grid spacing, with $360 \times 360$ and $480 \times 480$ grid points, respectively. The simulation was modified from the original Bill simulation so that its vertical levels and all model physics were all identical to those used in the hurricane nature run of Nolan et al. (2013). This simulation is also used in the study by Klotz and Nolan (2019) and is referred to as Bill2. As in Moon and Nolan (2015), the simulation reproduces the track and intensity of Hurricane Bill (2009) during this period remarkably well. At the time of interest, Bill was affected by the upper-level easterlies from the south and a trough to the west-northwest. This asymmetric environmental forcing enabled Bill to develop an outflow channel as well as a principal rainband.

\section{b. Overview of DMN17}

Figure 2 provides an overview of the control simulation (referred to as JET) of DMN17, with some snapshots of the westerly jet, outflow, and rainband at model times of $t=60,66$, and $72 \mathrm{~h}$. As the idealized vortex interacts with the prescribed westerly jet, a principal rainband forms in the northwest (NW) quadrant outside the primary eyewall around $t=66 \mathrm{~h}$ (Fig. 2f). Between 70 and $85 \mathrm{~h}$, the convection in the rainband moves cyclonically and gradually surrounds the TC eyewall (Fig. 2g). Finally, the secondary eyewall, which is the focus of DMN17, forms at around $t=85 \mathrm{~h}$. However, here we focus on the time around $t=66 \mathrm{~h}$, when the rainband is quasi stationary to the $\mathrm{NW}$ of the $\mathrm{TC}$. $\mathrm{Al}-$ though there also exists asymmetric convection outside the eyewall before $t=66 \mathrm{~h}$, the convection is not well organized and the outflow channel is relatively weak (shown later). Therefore, we choose $t=66 \mathrm{~h}$ as the time 
of interest in order to illustrate a strong signal of correlation between the outflow and the rainband. For comparison, we also consider the NOJET simulation from DMN17, where a TC develops in a quiescent environment that is identical to JET, but without the westerly jet. The snapshot of the NOJET simulation at $t=66 \mathrm{~h}$ is also shown in Figs. $2 \mathrm{~d}$ and $2 \mathrm{~h}$.

An enhanced upper-level outflow channel is evident from $t=60 \mathrm{~h}$ (Figs. 2a-c). The jet strength is also enhanced locally because of the TCEFI. Since the outflow has a horizontal range of about $2000 \mathrm{~km}$, it is much larger than the TC inner core, which is within a $300-\mathrm{km}$ radius as seen in the reflectivity field (bottom row of Fig. 2). We clarify here that the outflow in this study not only represents the synoptic-scale (approximately $2000 \mathrm{~km}$; spiral yellow and red shading below the jet in Figs. 2a-c) outflow channel, but also indicates the upper-level outward radial wind just outside the eyewall, with a scale of approximately $300 \mathrm{~km}$ (to be shown later). There is a principal rainband located outside the eyewall, and mostly concentrated within the $100<r<200-\mathrm{km}$ annulus. The rainband is quasi stationary in the NW quadrant of the TC before $t=72 \mathrm{~h}$ (Figs. 2e,f), after which the primary circulation becomes strong enough to move the rainband cyclonically (Fig. $2 \mathrm{~g}$ ). The upperlevel wind vectors near the inner core also show strong outflow in the NW quadrant (Figs. 2e-g), consistent with the outflow channel in the larger domain (Figs. 2a-c). The blue arrow in Fig. $2 \mathrm{f}$ represents the calculated VWS at that time. Although the value of VWS at $t=66 \mathrm{~h}$ is small at approximately $5.7 \mathrm{~m} \mathrm{~s}^{-1}$, the environmental flow already has a significant impact on the TC structure. In the NOJET simulation, the upper-level wind is quasi axisymmetric and confined (Fig. 2d) compared with the JET simulation. Also, there is no rainband outside the eyewall in the NOJET simulation (Fig. 2h).

Before moving to the discussion about the rainband and outflow relationship, we review the intensity change of the JET and NOJET simulations here, and briefly explore the TC intensity and outflow connections. Figure 3 compares the azimuthal-mean tangential wind at $z=2 \mathrm{~km}$ for the JET and NOJET simulations. Their main intensity difference starts at about $t=72 \mathrm{~h}$, after which the eyewall intensification in the JET simulation is much weaker. Furthermore, the wind field beyond the eyewall expands substantially farther outward in the JET simulation compared with the NOJET simulation. From that perspective, it appears as though the enhanced upper-level outflow might play a role in the weakened eyewall maximum intensity in the JET simulation, because of the enhanced convective activity and wind field expansion outside of the eyewall. Before $t=72 \mathrm{~h}$, although the radius of maximum azimuthal-mean
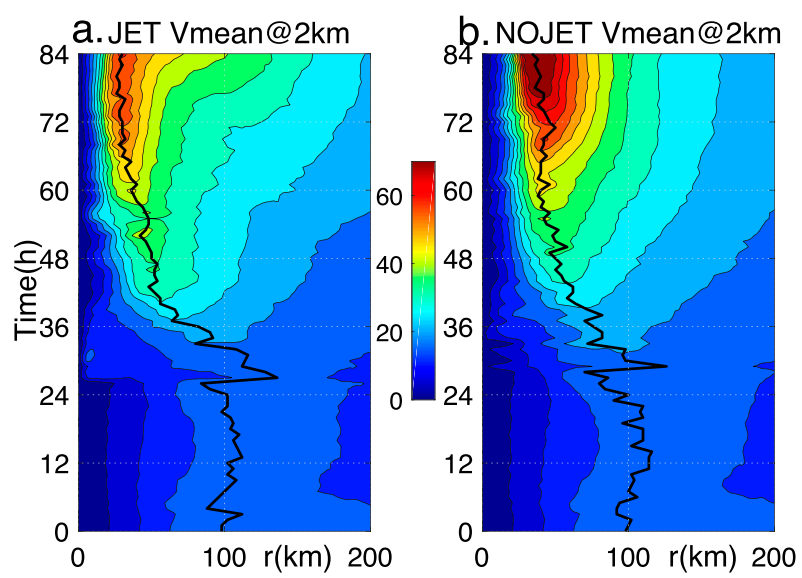

FIG. 3. Hovmöller diagrams of the azimuthal-mean tangential wind at $z=2 \mathrm{~km}$ for (a) JET and (b) NOJET simulations. Black contours indicate the radius of the maximum azimuthal-mean tangential wind at $z=2 \mathrm{~km}$.

tangential wind (RMW) at $z=2 \mathrm{~km}$ is slightly larger (black curve in Fig. 3) in NOJET than that of JET, we think they are going through similar stage of development. This is based on their similar wind strength and structure near the inner core, and their similar intensity from the time series of maximum wind speed in Fig. 7 of DMN17. Therefore, in the detailed structural comparison of the JET and NOJET simulations to be performed below, we assume that they are going through a similar intensity evolution at around $t=66 \mathrm{~h}$.

\section{Rainband and outflow relationship in idealized simulations}

\section{a. Vertical mass flux in the rainband and eyewall}

First, the vertical mass flux around the inner core (within $200 \mathrm{~km}$ of the center) is calculated using $t=63-69$-h composite-mean model data from DMN17. Figure 4a shows the vertical mass flux from the JET simulation, and the result from the NOJET simulation is presented in Fig. 4b for comparison. Here we assume that the inner-core upward mass flux all eventually goes into the outflow. To compare contributions from different regions to the outflow, we area integrate the vertical mass flux within each region. Three regions are displayed: 1) "eyewall," $0<r<100 \mathrm{~km}$ relative to the TC center; 2) "NW quadrant," $100<r<200-\mathrm{km}$ annular region, but only in the NW quadrant; and 3) "outer region," $100<r<200-\mathrm{km}$ annular region. From the definitions of the three regions, the area ratios of the eyewall to NW to outer annular are 1:0.75:3 (see the two circles in Figs. 2e-h). Our definition of the different regions is somewhat subjective: the eyewall slants outward, so it is not easy to differentiate between the eyewall 

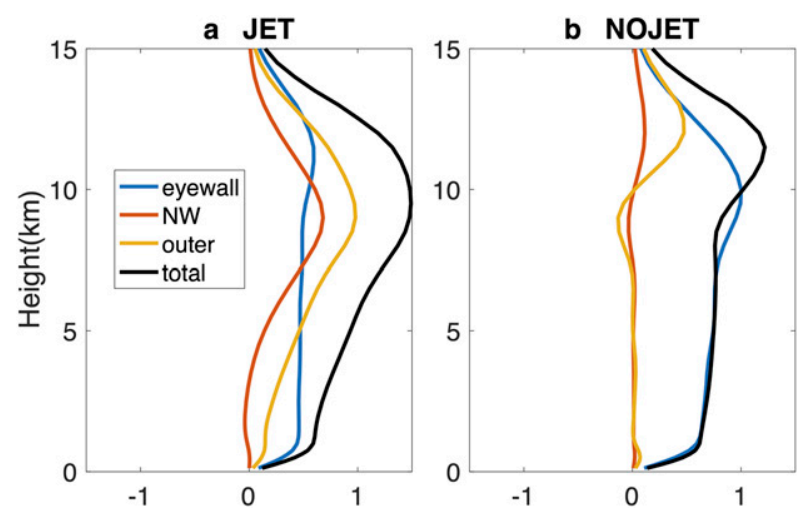

FIG. 4. (a) Area-integrated vertical mass flux in the JET simulation for $t=63-69 \mathrm{~h}$ composite mean in eyewall $(r<100 \mathrm{~km}$, blue curve), NW $(100<r<200 \mathrm{~km}$, but only at northwest quadrant, red curve), outer annulus ( $100<r<200 \mathrm{~km}$, brown curve), and total (eyewall plus outer annulus, black curve) regions. (b) As in (a), but in the NOJET simulation. All the data is normalized by the maximum of NOJET eyewall vertical mass flux.

region and outer region at upper levels. For example, in both Figs. $4 \mathrm{a}$ and $4 \mathrm{~b}$, the vertical mass flux at the eyewall region above $z=12 \mathrm{~km}$ is more or less similar to the outer region. Therefore, the vertical mass flux defined here in each region is more accurate below $z=12 \mathrm{~km}$ than above.

In the NOJET simulation in which the environment is quiescent, most of the upward mass flux is confined to the eyewall region (blue curve in Fig. 4b). The decrease of the vertical mass flux with height above $z=10 \mathrm{~km}$ indicates the divergent outflow in the upper levels. The outer region has a weak downward mass flux at around $z=9 \mathrm{~km}$ (orange curve in Fig. 4b), indicating subsidence. Note that all the data in Fig. 4 are normalized by the maximum of the NOJET eyewall vertical mass flux.

Compared with the NOJET simulation, the JET case shows a very different story. In the eyewall region (blue curve in Fig. 4a), the mass flux increases only a little with height compared with the NOJET simulation (blue curve in Fig. 4b), indicating that midlevel airmass convergence in the eyewall region is small. In the JET simulation, the outer annular region (orange curve in Fig. 4a) has a comparable or even larger upward mass flux than the eyewall region above $z=5 \mathrm{~km}$. The maximum of the outer annular vertical mass flux is located at approximately $z=9 \mathrm{~km}$, lower than the maximum of the eyewall vertical mass flux in the NOJET simulation $(>10 \mathrm{~km})$, probably suggesting a lower outflow layer above the rainband region compared with the eyewall region (to be described later).

Another interesting feature in the JET simulation is that the NW quadrant (rainband region; red curve in Fig. $4 a)$ is a major contributor to the outer annular upward mass flux at around $z=5-10 \mathrm{~km}$, especially when considering that the area of the NW quadrant is only one-quarter of that of the outer annulus. The maximum upward mass flux in the NW quadrant is also at a similar height as that of the outer annular counterpart. It is surprising to see that the upward mass flux in the rainband region exceeds the eyewall upward mass flux at approximately $z=7-10 \mathrm{~km}$. This finding is somewhat different from the traditional view, that the source of upper-level outflow mainly originates from the convective eyewall region, as in the NOJET simulation. Moreover, the contribution of the NW quadrant to the outer annular-region vertical mass flux illustrates the azimuthally asymmetric feature of TC dynamics.

Last, the eyewall upward mass flux in the JET simulation is generally smaller than in the NOJET simulation, because the rainband in the JET simulation consumes a significant portion of the low-level inflow coming from outside the TC. In contrast, the total (eyewall plus outer annular region) vertical mass flux in the JET simulation (black curve in Fig. 4a) is larger than that in the NOJET one (black curve in Fig. 4b), consistent with the strong outflow in the JET simulation.

\section{b. Vertical secondary circulation}

Based on the above vertical mass flux calculation, we found that the rainband region is an important source for outflow, but what is the vertical structure of the rainband and outflow? To address this question, we examine the azimuthally asymmetric velocity structures via vertical cross sections of the radial and vertical wind through the downshear-left direction of the vortex in the JET simulation at $t=66 \mathrm{~h}$ (Fig. 5a). The cross section is from southeast (SE) to NW, depicted by the black line in Fig. 2f. The outflow is indicated by the outward radial wind at $z=12-14 \mathrm{~km}$, with a range of $300 \mathrm{~km}$ radially. The corresponding result from the NOJET simulation is also presented for comparison (Fig. 5b).

Consistent with Jones (1995), Reasor et al. (2004), and Riemer et al. (2010), who used the vortex tilt induced by the VWS to explain the convective asymmetry around the inner core, this SE-NW (downshear left) direction has larger tilt compared with other directions in the JET simulation (not shown). It is noteworthy that while the outer vortex (outside the eyewall) is tilted to the downshear left, the eyewall-scale vortex is not tilted as much (see the eyewall vertical velocity field in Fig. 5a), also consistent with previous studies. However, unlike Riemer et al. (2010), who argued that the wavenumber-1 asymmetry of relative vorticity was important in inducing the asymmetric convection, there is only a very weak wavenumber-1 vorticity anomaly in our case (not shown). We suggest that this is because the VWS value 


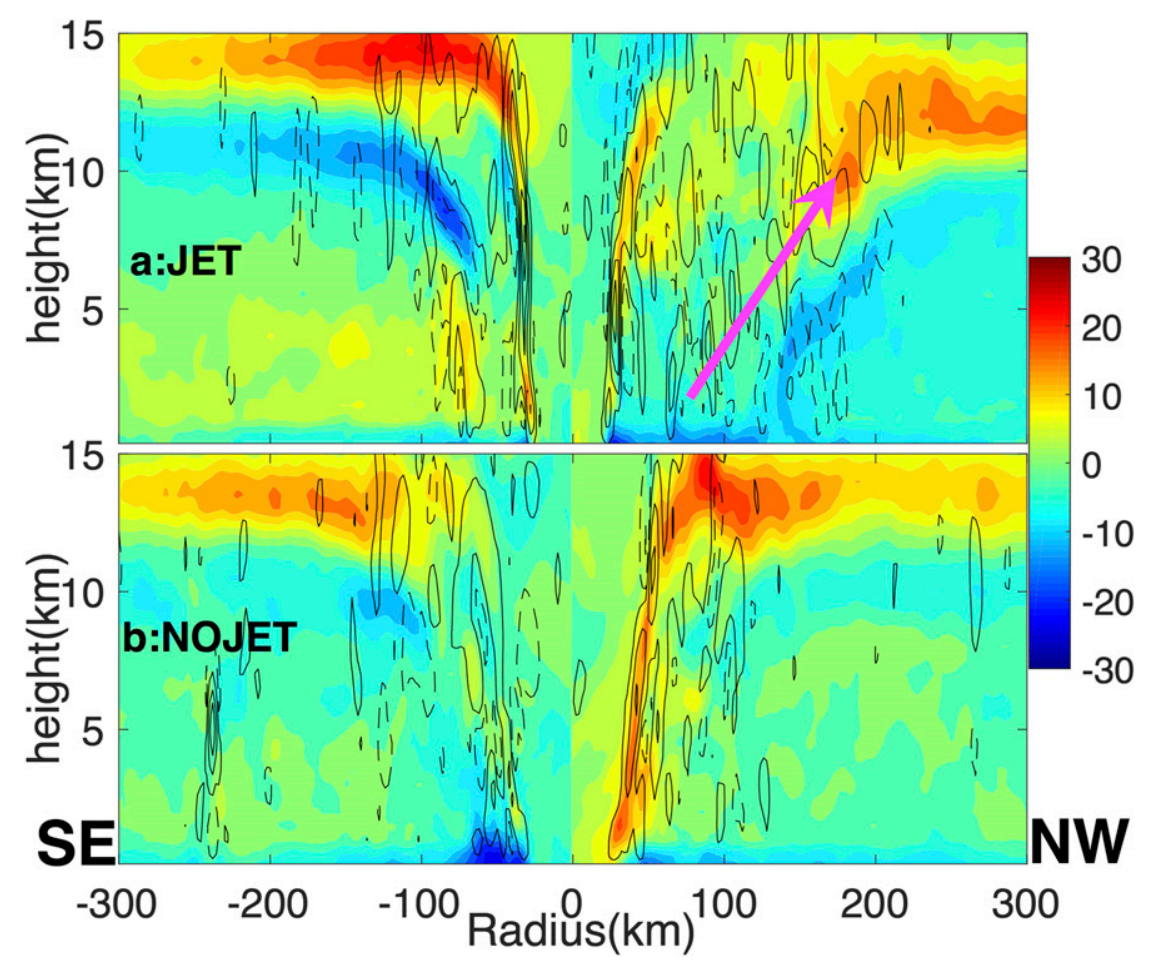

FIG. 5. (a) The vertical cross section made through the "downshear left" direction in the JET simulation at $t=66 \mathrm{~h}$, from SE (left) to NW (right), as indicated by the black line in Fig. 2f. The color shading shows radial wind and the contours show vertical velocity (dashed contour indicate downdrafts). The big magenta arrow indicates the secondary circulation related to the rainband. (b) As in (a), but for the NOJET simulation.

$\left(5.7 \mathrm{~m} \mathrm{~s}^{-1}\right)$ here is much smaller compared to the $15 \mathrm{~m} \mathrm{~s}^{-1}$ used by Riemer et al. (2010).

In Fig. 5a, the upper-level outward radial wind maximum is farther away from the eye in the NW quadrant than in the SE quadrant, clearly associated with the larger-scale asymmetric outflow channel. The eyewall outflow is weakened correspondingly on the NW side. It is interesting that the outflow height in the SE quadrant $(\sim 14 \mathrm{~km})$ is higher than that in the NW quadrant $(\sim 12 \mathrm{~km})$. The relatively low outflow height in NW is perhaps related to the environmental flow, as the westerly jet center is prescribed to be at $z=12 \mathrm{~km}$. In contrast, the SE quadrant is not affected much by the jet, since the outflow height in the NOJET simulation is also around $14 \mathrm{~km}$ (Fig. 5b). In addition, the vertical motion corresponds quite well with the radial wind, in that stronger updrafts are located along with the outward radial wind outside the eyewall in the NW quadrant. The radial wind together with the upward motion indicates that another secondary circulation (indicated by the magenta arrow in Fig. 5a) is created outside the eyewall on the NW side, associated with the asymmetric outflow. In contrast, for the NOJET simulation, the SE and NW sides are quite similar compared with the JET simulation, especially the location of the maximum updraft and upper-level outflow. Some asymmetry is present in the NOJET simulation, because the eyewall is still developing and not steady yet at this time (see the generally symmetric but slightly broken eyewall in Fig. 2h). Also, there is no indication of a secondary circulation outside the eyewall in the NOJET simulation.

The equivalent potential temperature $\theta_{e}$ is a conserved quantity following a parcel in the atmosphere with moist adiabatic processes. If the $\theta_{e}$ in the rainband region is similar to that of the outflow region, it might be helpful to support our argument that the rainband feeds the outflow. Figure 6 shows the vertical cross section of $\theta_{e}$ in the NW direction in both JET and NOJET simulations. The light green shading region $\left(348<\theta_{e}<350 \mathrm{~K}\right)$, bounded by the bolded red contour, is the value of $\theta_{e}$ we use to represent that of the outflow layer. In addition to the eyewall region $(r<50 \mathrm{~km})$, the tilted rainband region (starting from $r \sim 100 \mathrm{~km}$, indicated by the upward vertical velocity; also indicated by the magenta arrow in Fig. 5a) in the JET simulation contains the same $\theta_{e}$ value as that of the outflow region. This is in contrast to the NOJET simulation, where the eyewall $\theta_{e}$ becomes the only source for the outflow $\theta_{e}$. That again proves that 

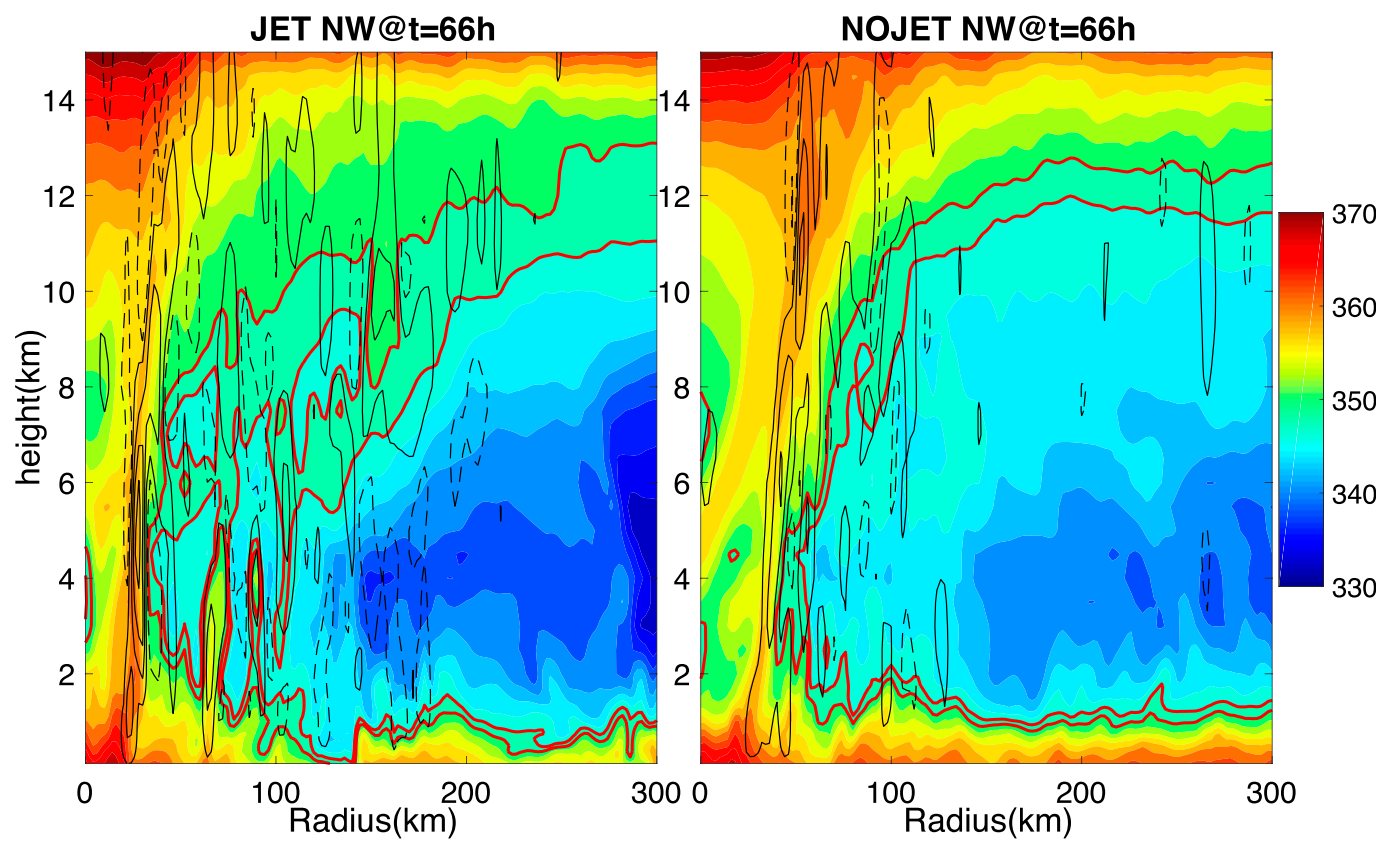

FIG. 6. The northwest vertical cross section of equivalent potential temperature $\theta_{e}$ (shading) of (left) JET and (right) NOJET simulation at $t=66 \mathrm{~h}$. The red bolded contour indicates $\theta_{e}$ values between 348 and $350 \mathrm{~K}$, which are the values of outflow layer in the JET simulation. Solid (dashed) black contours indicate updrafts (downdrafts), as in Fig. 5.

the rainband can be an important source of the outflow jet under the environmental flow.

\section{c. Trajectory analysis}

Can air parcels travel directly from the rainband region upward to the outflow? Here we use forward and backward trajectory analyses (Fig. 7) to visualize the process. For the forward trajectories, the initial horizontal locations are specified within the rainband region: $x=[-150,-100] \mathrm{km}, y=[100,150] \mathrm{km}$, with an interval of $10 \mathrm{~km}$ (small brown box in Fig. 7, top center). The initial height is at $z=7 \mathrm{~km}$, high enough to capture both the convective and stratiform updrafts, and low enough to avoid the outflow. For backward trajectories, the final locations are specified at $x=[-200,-150] \mathrm{km}, y=[150$, 200] $\mathrm{km}$, again with an interval of $10 \mathrm{~km}$ (small green box in Fig. 7, bottom center), and $z=12 \mathrm{~km}$. This is the upper-level outflow region, and the purpose here is to diagnose where the source of the outflow comes from. We have also made 121 trajectories (instead of 36 here) with a horizontal resolution of $5 \mathrm{~km}$ in the same region, and found similar results. To show the trajectories clearly, we only present the 36-trajectory analysis below.

For forward trajectories (top panels of Fig. 7), the parcels can be separated into three groups. (i) A small fraction of parcels (3 out of 36) follow the convection and go directly upward to the outflow region (the colored contours). (ii) Some parcels rise slowly and follow the cyclonic winds, and finally approaches the outflow region. These parcels seem to be related to the stratiform updrafts in the middle to upper levels of the rainband region. (iii) Other parcels sink slightly, and then follow the cyclonic winds. Not surprisingly, the motion of the parcels is mainly controlled by the TC primary circulation (center panels of Fig. 7b). Although the number of convective parcels that directly enter the outflow is small (the first group), we note that undiluted convective plumes rarely exist in the upper tropical troposphere (Romps and Kuang 2010). We therefore suggest that the second group of parcels in the rainband that are carried by slow updrafts (and are diluted) might be the main contributor to the outflow.

For backward trajectories (bottom panels of Fig. 7), it is clear in the $x-z$ cross section that only a small portion of parcels originate directly from the low-level boundary layer eyewall region. This small number of parcels that travel directly from the eyewall into the outflow indicates that, under the influence of the environmental flow, parcels outside the eyewall might also be an important source of the upper-level outflow. Many other parcels come from different heights through the troposphere and meander for a while before going upward. Those parcels from different heights with slow upward motion resemble the second group of parcels from the forward analysis described above, and might also originate from the rainband region. There are also signals of 

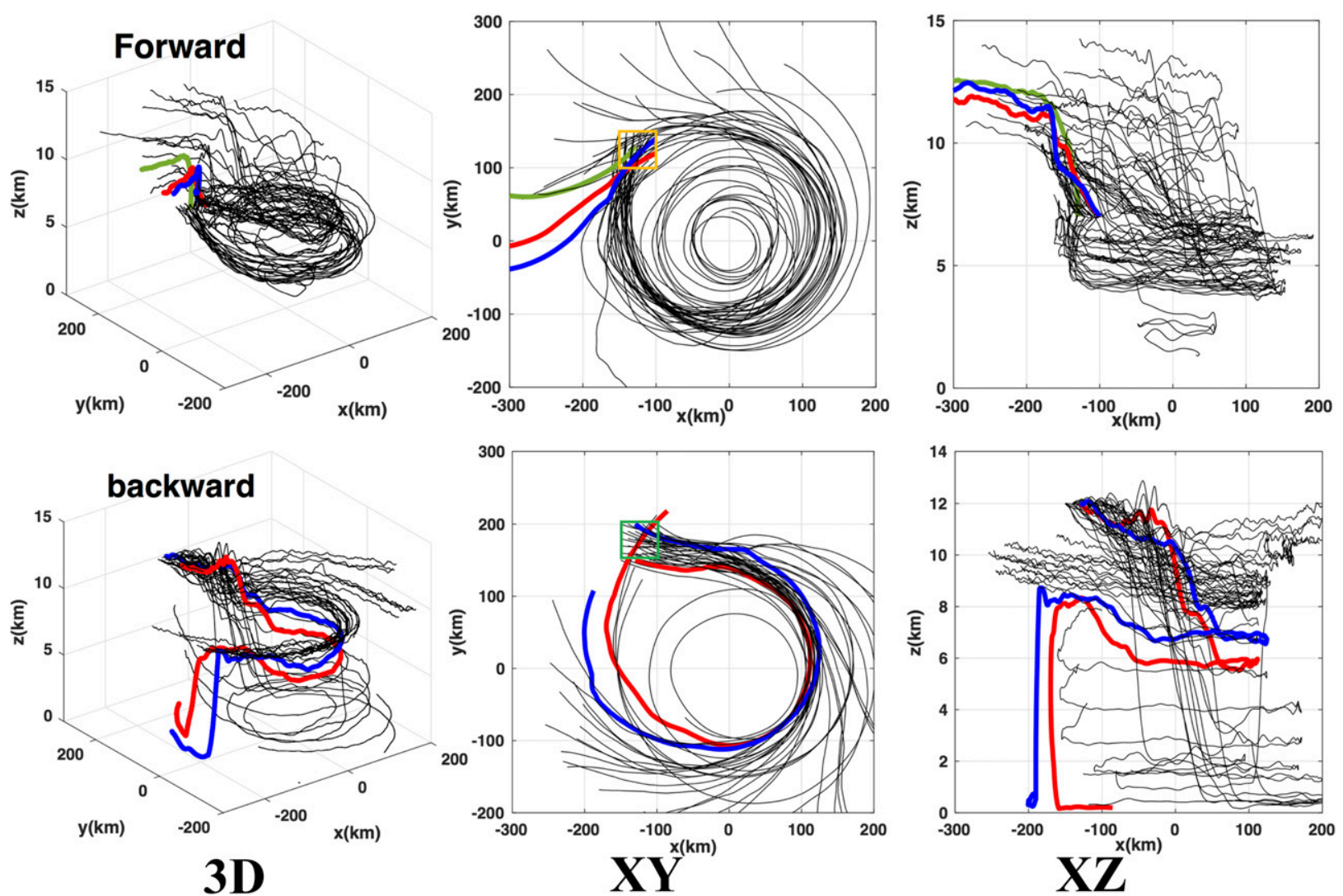

FIG. 7. (top) Forward trajectories at $t=59-71 \mathrm{~h}$ in JET simulation viewed in (left to right) $3 \mathrm{D}, X-Y$, and $X-Z$ projections. (bottom) As in the top panels, but for backward trajectories. The bolded contours indicate trajectories that are located at the convective region in the rainband. Little boxes in the center panels indicate the initial parcel location.

rainband convection (two colored contours in the bottom panels of Fig. 7): they originate from the boundary layer rainband region, and go directly upward to about $z=8-9 \mathrm{~km}$ before rising slowly and cyclonically to the outflow.

Since both the forward and backward trajectories demonstrate that parcels in the rainband region are able to propagate into the upper-level outflow, through either convective or stratiform updrafts, we again deduce that the rainband region is an important contributor to the outflow.

\section{d. Damped rainband experiment}

Can the upper-level outflow be weakened when the rainband is weakened, if the outflow-rainband relationship as described above is true? To address this question, we conduct a sensitivity experiment in which the rainband is damped. At $t=65 \mathrm{~h}$ in the JET simulation, we reduce the water vapor mixing ratio (QVAPOR in the WRF output) in the rainband region to 0 , so that the convection is quickly suppressed. We then rerun the simulation for another $1 \mathrm{~h}$ to investigate how the outflow responds. The damped rainband region is prescribed from the northeast (NE) cyclonically to the west, within an annulus of $100<r<200 \mathrm{~km}$ (the region within the white line in Figs. 8a and 8d). We also note that in the damped rainband experiment (DR) simulation, except for the water vapor mixing ratio in the rainband, everything else is exactly the same as the control (JET) simulation in DMN17.

Just $1 \mathrm{~h}$ after the damping, most of the convection within the rainband has dissipated (Fig. 8d) compared with the initial condition at $t=65 \mathrm{~h}$ (Fig. 8a). A comparison of the NW vertical cross section between $1 \mathrm{~h}$ after DR and initial condition at $t=65 \mathrm{~h}$ shows that the upper-level radial wind outside the eyewall in DR is weakened from 15 to $10 \mathrm{~m} \mathrm{~s}^{-1}$ approximately, while the radial wind close to the eyewall is enhanced a bit (Figs. $8 \mathrm{~b}, \mathrm{e})$. Interestingly, with the damping of the rainband, the low-level inflow near the eyewall in DR simulation is enhanced, consistent with previous studies, (e.g., Bister and Emanuel 1997; Wang 2009) which suggested that the outer rainband could impede the intensification of the eyewall. Furthermore, there is no organized updraft (or secondary circulation) in the rainband region in the DR simulation (Fig. 8e). We have also compared the vertical mass flux before and after damping (Figs. 8c,f). It is clear 

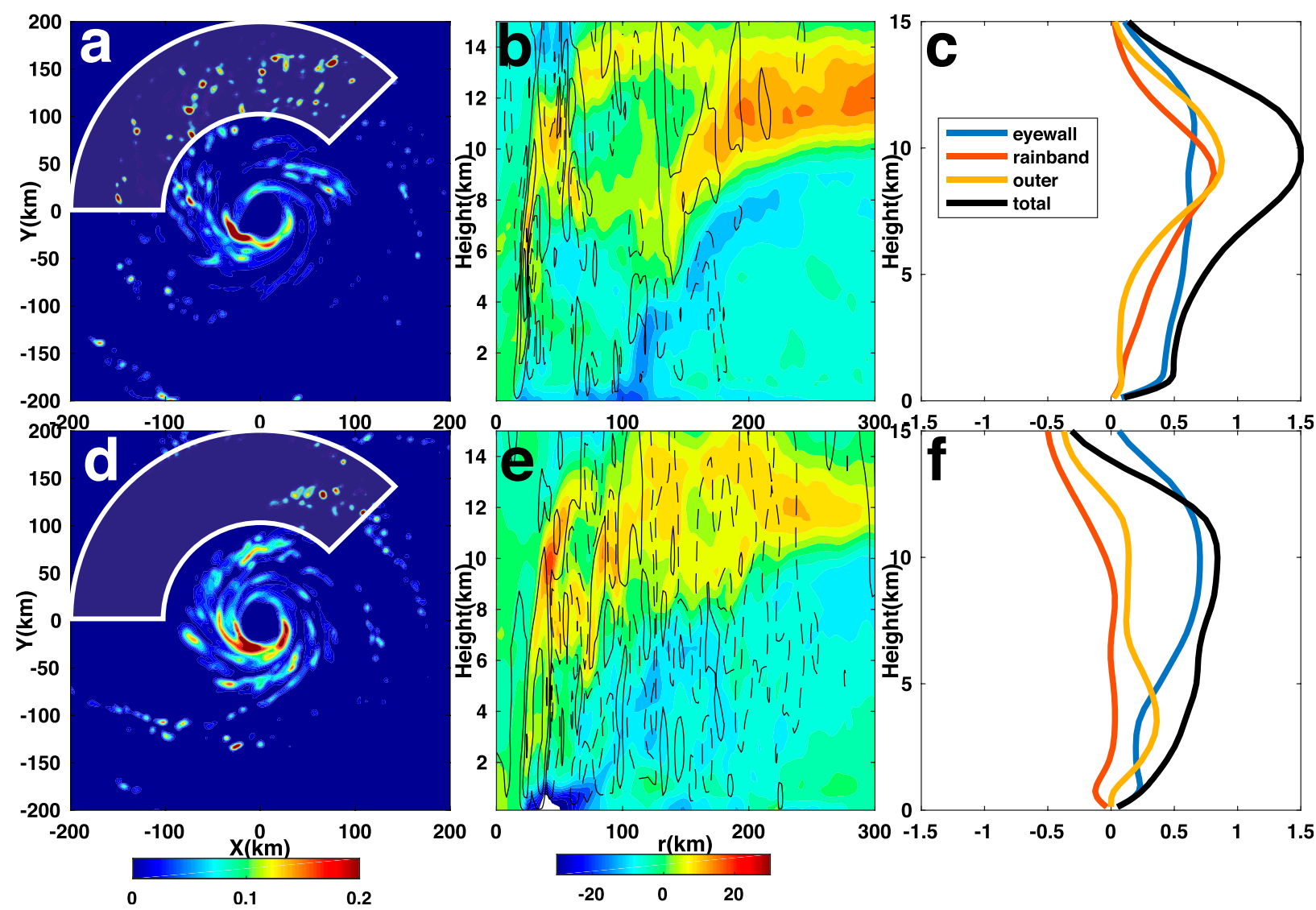

FIG. 8. (a) Rain rate (mm per time step) at $t=65 \mathrm{~h}$ in the JET simulation, which is also the initial condition for the "damped rainband" (DR) simulation. White curve indicates the damped region. (b) Vertical cross section in the NW direction showing radial wind (shaded) and vertical velocity (contours at $-4.5,-2.5,-0.5,0.5,2.5$, and $4.5 \mathrm{~m} \mathrm{~s}^{-1}$; dashed contours indicate downdrafts) for the control (JET) simulation at $t=65 \mathrm{~h}$. (c) Vertical mass flux of the JET simulation at $t=65 \mathrm{~h}$. Note that the "rainband" indicates the damped region. (d)-(f) As in (a)-(c), respectively, but from $1 \mathrm{~h}$ after the DR simulation.

that the rainband region has little positive vertical mass flux after 1-h damping (Fig. 8f).

We therefore suggest that the upper-level outflow and the outer rainband are directly related, with a weakened outflow (by approximately $5 \mathrm{~m} \mathrm{~s}^{-1}$ ) responding to the damped rainband. This point is also supported by the idealized simulation of Shi et al. (1990). Instead of damping the rainband, Shi et al. (1990) numerically enhanced the upper-level outflow outside the inner core $(r>500 \mathrm{~km})$. They found that the convection was induced locally below the enhanced outflow outside the inner core. Thus, the enhanced outflow channel is directly linked with the principal outer rainband as in the JET simulation, and the rainband can be a local source of the atmosphere to the TCEFI-enhanced outflow channel.

\section{e. Mechanism behind the rainband and outflow relationship}

Although the above subsections illustrate a direct connection between the upper-level outflow and asymmetric rainband region, it is still not clear how such a connection is built. Is the outflow channel created by the TCEFI first, then inducing a rainband, or vice versa? It is difficult to answer that question by just comparing the time evolutions of the outflow and rainband, because convection connects the rainband with the upper-level outflow so quickly that they evolve nearly simultaneously. Figure 9 shows the early stages (before $t=60 \mathrm{~h}$ ) of the JET simulation. Because of the advection of the westerly environmental flow in the north and weak TC primary circulation, the asymmetric convection outside the eyewall is mainly located within the NE quadrant, before being organized into a quasi-stationary rainband in the NW quadrant (Fig. 2). While both the TC inner core and outflow are evolving quickly at $t=36-54 \mathrm{~h}$, it is hard to identify which is leading which. We therefore suggest that the convection outside the eyewall and the outflow channel are closely coupled to each other.

It might appear that the enhanced outflow is inducing the outer rainband, because the environmental flow is 

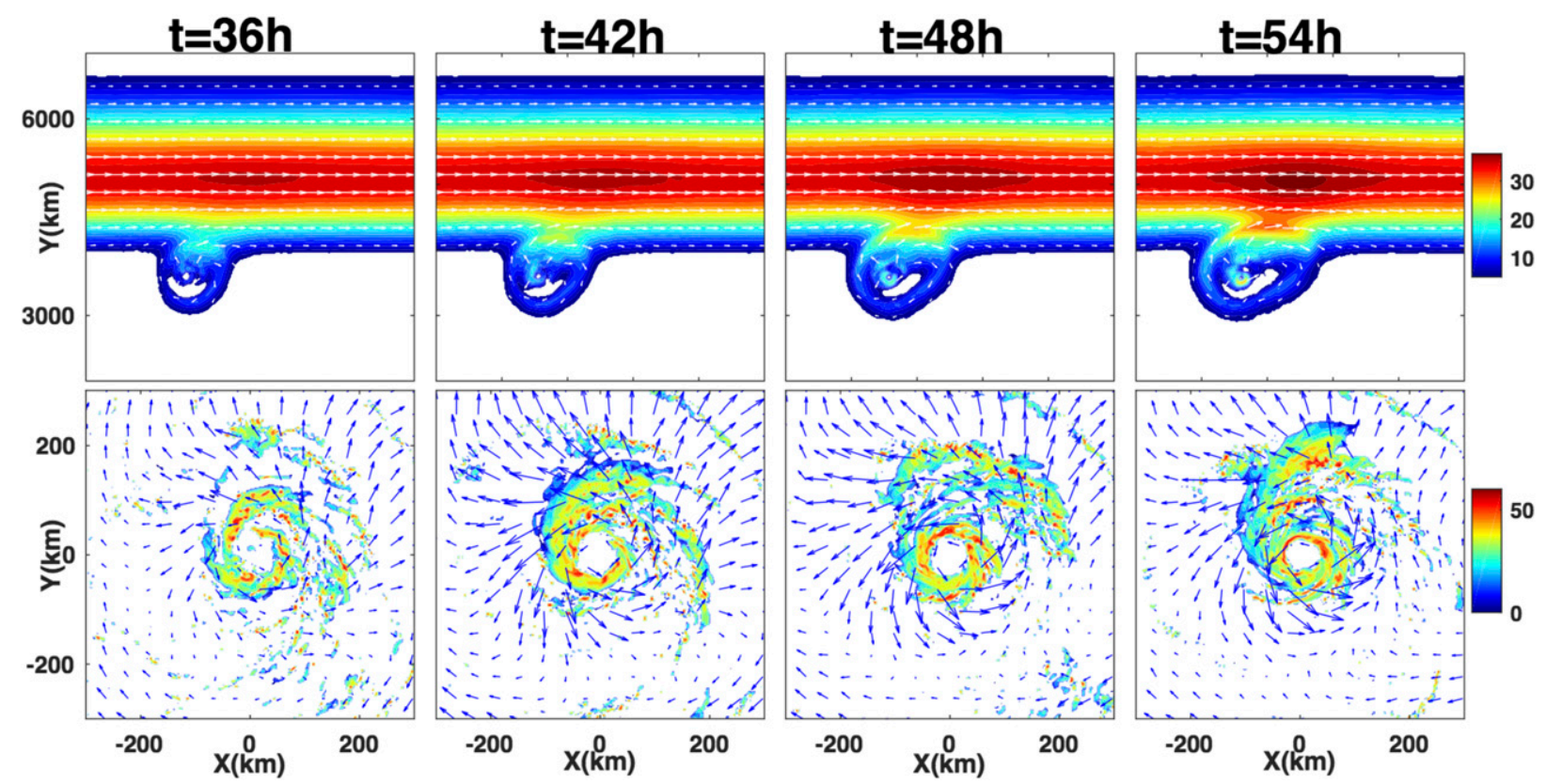

FIG. 9. As in Fig. 2, but for (left to right) $t=36,42$, 48, and $54 \mathrm{~h}$ in the control (JET) simulation.

introduced in the upper troposphere in our simulation. However, a detailed analysis suggests that it is not the case. We hypothesize here that the upper-level enhanced outflow and outer rainband are coupled features and occur simultaneously. Both of them belong to the TC response to the upper-level environmental flow.

We first use the inertial stability to clarify why the enhanced outflow is concentrated within the NW quadrant in the JET simulation. The inertial stability is defined as $I=(f+2 V / r)(f+\zeta)$, where $V$ is the tangential wind, $r$ the distance to the TC center, $f$ the Coriolis parameter, and $\zeta$ is the vertical component of relative vorticity. In the anticyclonic outflow region, $I$ is usually negative or slightly positive. When imposed by the westerly jet, the northern (southern) part of the TC "feels" anticyclonic (cyclonic) wind. Therefore, the northern part of the TC is more inertially unstable than the southern part (see the light yellow and green region of the JET simulation in Fig. 10). As a consequence, the upper-level outflow is concentrated on the asymmetric inertially unstable (or weakly stable) region. As a comparison, in the NOJET simulation (right panel of Fig. 10) the inertially unstable region is quite symmetrical, and that appears to be why no asymmetric outflow channel is formed in the NOJET simulation. Molinari and Vollaro (2014) observed the inertial/symmetric instability in the outflow layer of Hurricane Ivan (2004) using dropwindsonde data. A similar instability is also found in the hurricane nature run simulation of Nolan et al. (2013) (see Fig. 6 in Molinari and Vollaro 2014).
Therefore, although symmetric TCs have some inertial instability in the azimuthal mean, the sheared TCs might have larger instability in the outflow quadrant, similar to the asymmetric inertial instability argued by Stevens and Ciesielski (1986).

More interestingly, we have found a feature that is able to connect the rainband and outflow: a descending inflow (or radially inward downdraft). Focusing on the NW part of Fig. 5a, just below the enhanced outflow, a descending inflow starts at about $z=10 \mathrm{~km}$ and extends all the way down to the boundary layer. This descending inflow is reminiscent of the findings by Didlake et al. (2018), that a descending inflow was located in the outer region of Hurricane Earl's (2010) primary rainband. Note that Earl's inflow starts at about $z=6 \mathrm{~km}$, not as high as the inflow in our JET simulation. One potential reason for this is the limitations of radar observations. This descending inflow is also a persistent feature (similar to the enhanced outflow and rainband): it even occurs in the very early stage, for example, as early as at $t=42 \mathrm{~h}$, when the outer rainband starts to form. We show the horizontal feature of the descending inflow in Fig. 11 at three different heights and at $t=66$ and $42 \mathrm{~h}$, respectively. To show descending inflow clearly, we use solid contour in Fig. 11 to represent downdraft, while the wind vector indicates radial wind. The shading in Fig. 11 is diabatic heating rate. It is clear that the diabatic cooling (blue shading in Fig. 11) corresponds very well with the downdraft (contours in Fig. 11). Actually, the diabatic heating field looks more like a smoothed field of 

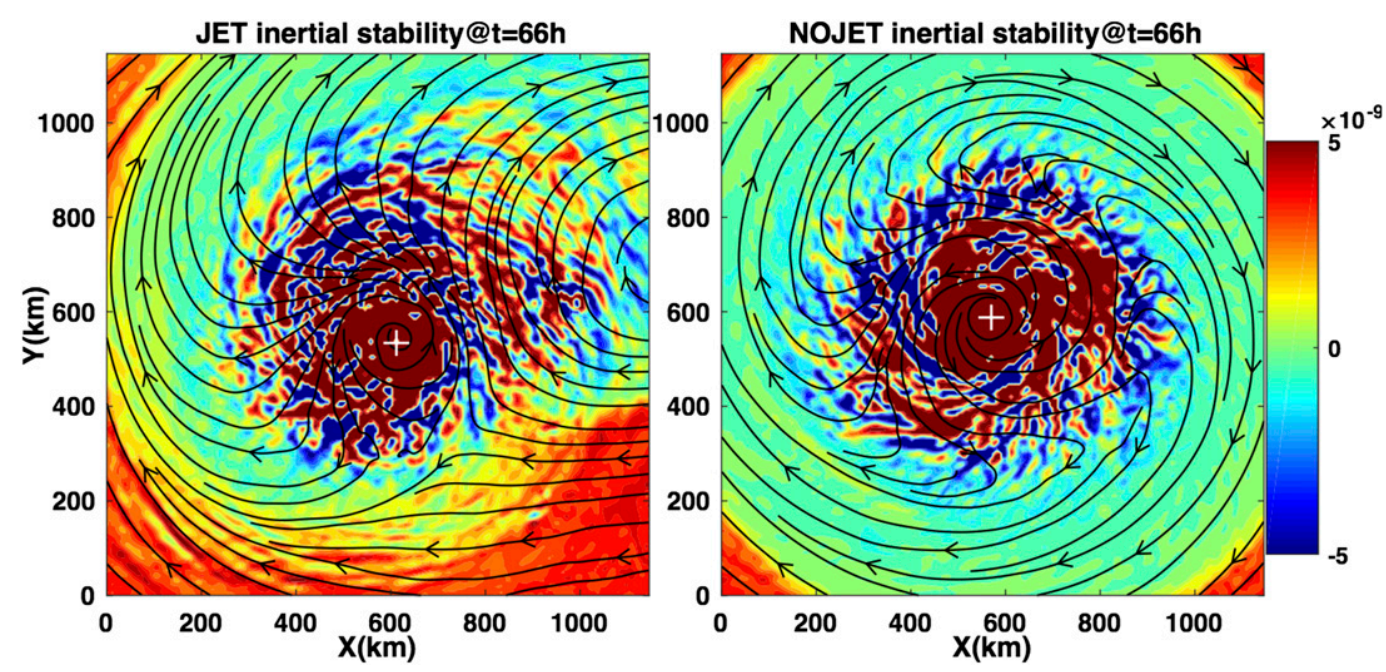

FIG. 10. Horizontal view of inertial stability ( $\mathrm{s}^{-2}$, shaded) at $z=12 \mathrm{~km}$ for (left) JET and (right) NOJET simulations at $t=66 \mathrm{~h}$. Streamlines indicate wind direction.

vertical velocity, and that is why we choose diabatic heating as the shading in Fig. 11. The descending inflow is quite visible in the upper level (Fig. 11a): it spirals cyclonically inward from the NW quadrant to the SE quadrant (blue shading in Fig. 11a). Therefore, Fig. 11a demonstrates that the descending inflow in the upperlevel SE quadrant in Fig. 5a belongs to the same feature as the NW descending inflow in Fig. 5a. At lower levels (Fig. 11c), a line of descending inflow is less clear than at upper levels (remember that the environmental flow is small in the TC inner core, such that the VWS is only about $5.7 \mathrm{~m} \mathrm{~s}^{-1}$ at $t=66 \mathrm{~h}$ ). However, it does the job of affecting the boundary layer dynamic and thermodynamic conditions, so that the rainband is located to the right side of the descending inflow. We do find in our additional numerical experiments (not shown) that, under stronger environmental flow, the descending inflow is a much clearer feature even in the boundary layer. At $t=42 \mathrm{~h}$, the descending inflow is slightly weaker than that of at $t=66 \mathrm{~h}$, consistent with the much weaker outflow and rainband (see Fig. 9). Nevertheless, the descending inflow is still an important mesoscale feature, and is the key to bridging the upper-level outflow and outer rainband. We therefore suggest that the formation of the descending inflow starts as a result of the dynamical response of the enhanced outflow aloft, and is then supplemented by the subsequent diabatic cooling of liquid (or solid) droplets. The location of the descending inflow defines a lateral boundary where the boundary layer inflow can converge and help the development of the outer rainband, which in turn provides an air source to the upper-level enhanced outflow. Although a fully clear picture needs further study, we believe that the enhanced upper-level outflow, descending inflow, and outer rainband are all coupled features that concurrently form the TC response to the upper-level environmental flow. We suggest that the descending inflow is also strongly related to TC intensity change, and that it deserves further investigation with systematic modeling and analysis.

\section{Results from a simulation of Hurricane Bill (2009)}

In our idealized framework, the westerly jet is the only environmental flow that contributes to the enhanced outflow channel. Are the same processes seen in realistic situations? We expect that any asymmetric environmental flow should work for our hypothesis. Here, we use a simulation of Hurricane Bill (2009) to explore the connection between rainbands and outflow channels in a realistic setting. A detailed description of this simulation is in section 2. At the time of interest $(t=0600$ UTC 20 August 2009), the real Bill was near its maximum intensity and possessed a principal rainband in the NE quadrant, whose radial distance to the TC center was about $250 \mathrm{~km}$ (Fig. 12c). At the same time, the upperlevel outflow was evident in both the AMVs (Fig. 12a) and reanalysis data (Fig. 12b). This outflow channel starts from above the rainband and rotates anticyclonically to the south, remaining coherent out to a distance of more than $2000 \mathrm{~km}$ from the TC center. There is also an upper-level trough to the west-northwest of Bill, guiding the northern part of the outflow channel. We suggest that this upper-level trough, together with the strong upper-level easterlies in the deep tropics, contributed to Bill's outflow channel (Fig. 12a). The 200-hPa horizontal wind speed in the Bill2 simulation 

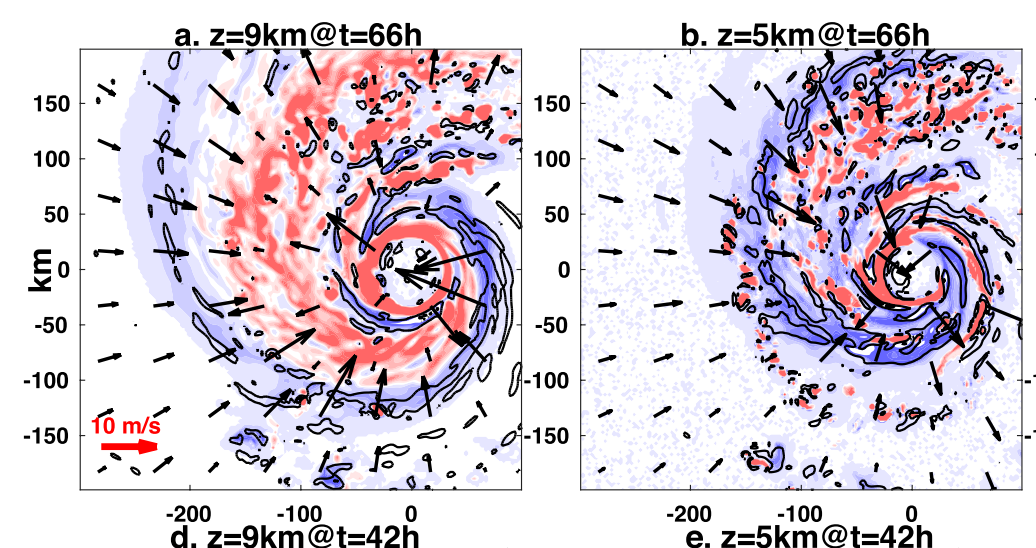

b. $z=5 \mathrm{~km} @ \mathrm{t}=66 \mathrm{~h}$
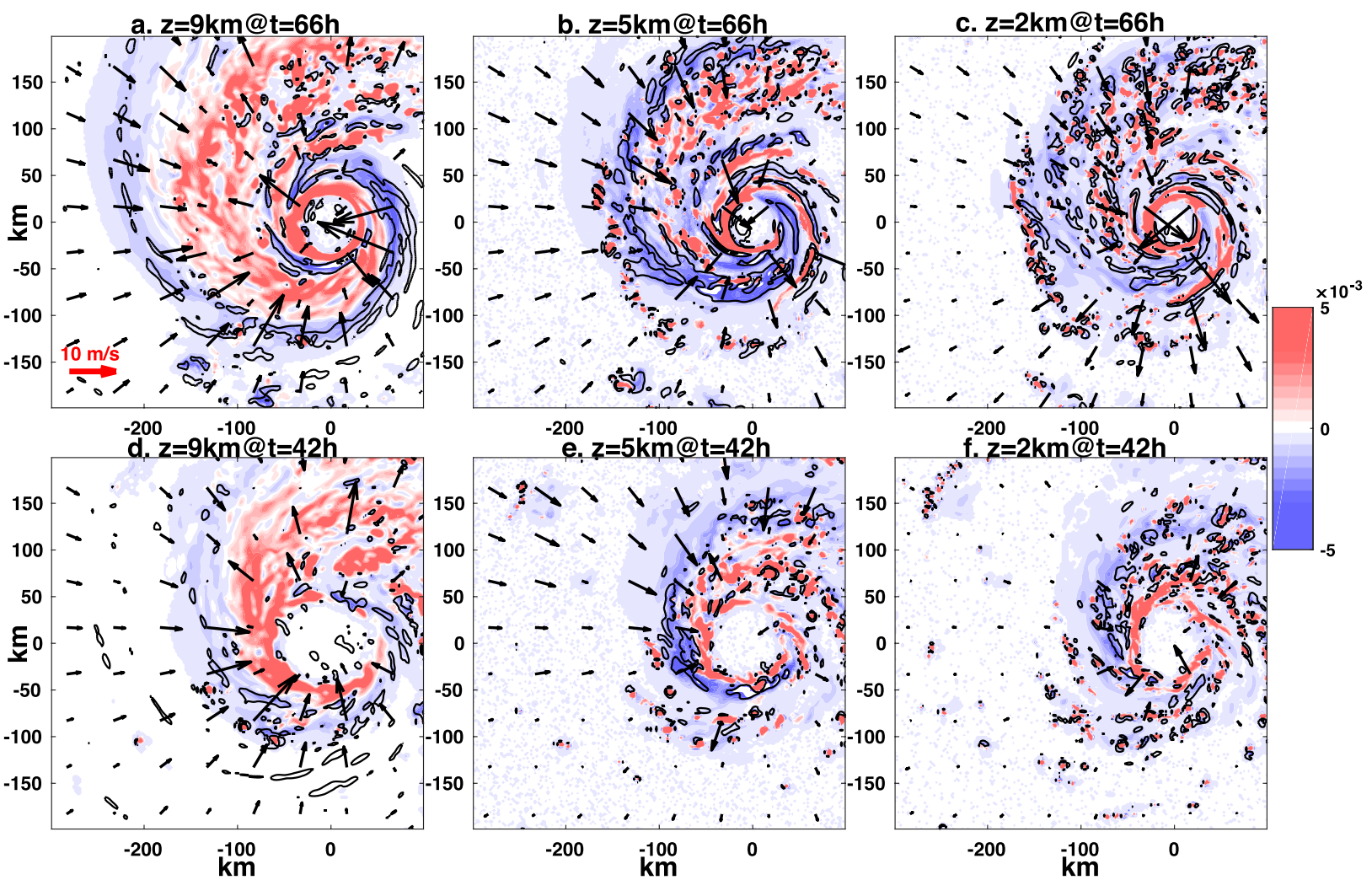

FIG. 11. The radial wind (vectors), downdraft (contours at $-8.5,-4.5,-0.5 \mathrm{~m} \mathrm{~s}^{-1}$ ), and diabatic heating rate (K s${ }^{-1}$, shading) in the JET simulation at $t=66 \mathrm{~h}$ for $z=$ (a) 9 , (b) 5 , and (c) $2 \mathrm{~km}$. (d)-(f) As in (a)-(c), but at $t=42 \mathrm{~h}$.

also confirms that the outflow channel is the dominant feature at that time (Fig. 12d).

The lower-level simulated reflectivity and upper-level streamlines of Bill2 are shown in Fig. 13a. It is clear that the outer rainband and enhanced outflow are both in the NE quadrant. Although another line of convection is located to west of Bill2, that is shallow convection that is not related to the upper-level outflow. The vertical mass flux of Bill2 is also shown in Fig. 13b. Here, we use a slightly different method to calculate Bill2's vertical mass flux, that we vary the eyewall region from $r=40 \mathrm{~km}$ at $z=0 \mathrm{~km}$ to $r=70 \mathrm{~km}$ at $z=15 \mathrm{~km}$ linearly. This is because the eye in Bill2 is compact, and the rainband region is very close to the eyewall in NE quadrant (Fig. 13a). Using a constant eyewall radius will result in a very small vertical mass flux in the eyewall in the upper level. The outer annular region has a similar upward mass flux compared with the eyewall. In addition, the NE quadrant, where the rainband is concentrated, contributes about $50 \%$ of the total outer annular flux, despite the fact that it is only one quarter of the area of the outer annulus. This is consistent with our idealized JET simulation, which demonstrated that the outer rainband was an important source of the upper-level outflow (Fig. 4a). Readers may find that, different from our JET simulation, the largest vertical mass flux in Bill2 is at $z=6 \mathrm{~km}$. To answer why, we analyzed the horizontal wind in the midlevel, and found that Bill2 was situated within a relatively strong $\left(\sim 10 \mathrm{~m} \mathrm{~s}^{-1}\right)$ southerly midlevel steering flow. We can see some of the steering flow in Fig. 13c, where the radial flow is generally positive between $z=4-8 \mathrm{~km}$ in the north-northeast direction. Furthermore, we also found a weak descending inflow below the enhanced outflow in the cross section of north-northeast direction (Fig. 13c). That descending inflow might help the organization and location of the rainband. Therefore, although Bill encountered a much more complicated environmental wind field than the idealized westerly jet, the location of the outer rainband and upper-level outflow, the vertical mass flux, and the descending inflow below the outflow all support the idea that the rainband and enhanced outflow connect directly through the TC response to the environmental flow.

\section{Sensitivity experiments}

One question remains to be clarified: Does the outflowrainband relationship always hold if the environmental flow or initial TC intensity and structure is different? To 

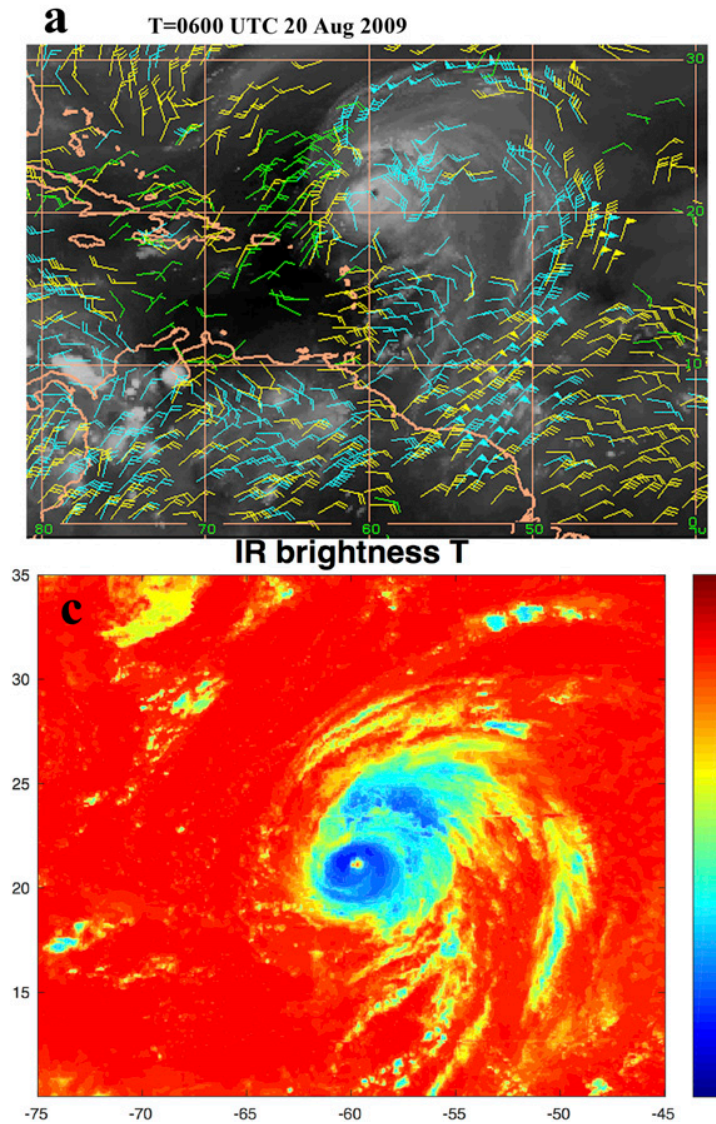

FIG. 12. (a) Hurricane Bill (2009) mid- to upper-level AMVs and water vapor satellite image at 0600 UTC 20 Aug 2009. (b) ERA-Interim-derived 200-hPa wind speed (shaded) plus wind vectors of Hurricane Bill. (c) Infrared brightness temperature of Bill. (d) As in (b), except using data from simulation of Bill (see section 2 for Bill simulation details).

make the outflow-rainband relationship more general, six sensitivity experiments named S1-S6 are conducted. We note that the basic model setup is nearly identical to the JET control simulation, except that here we use WRF single-momentum 6-class microphysics (WSM6; Hong and Lim 2006) instead of WSM5 (used in JET control simulation) to include more microphysics (such as graupel). A comparison (not shown) indicates that these two microphysics schemes result in very similar patterns of convection or stratiform rainfall. Other than the microphysics scheme, S1 is identical to the JET control simulation except for the environmental flow, which is in uniform westerly shear (no meridional variation). The value of the VWS in S1 is approximately $3 \mathrm{~m} \mathrm{~s}^{-1}$, same as the calculated VWS of the initial JET simulation. In S2, an upper-level trough is embedded in the westerly jet to simulate the midlatitude trough. The trough is created by adding a Gaussian vorticity anomaly into the westerly jet. The initial TC vortex is first integrated in the quiescent environment for $72 \mathrm{~h}$ to

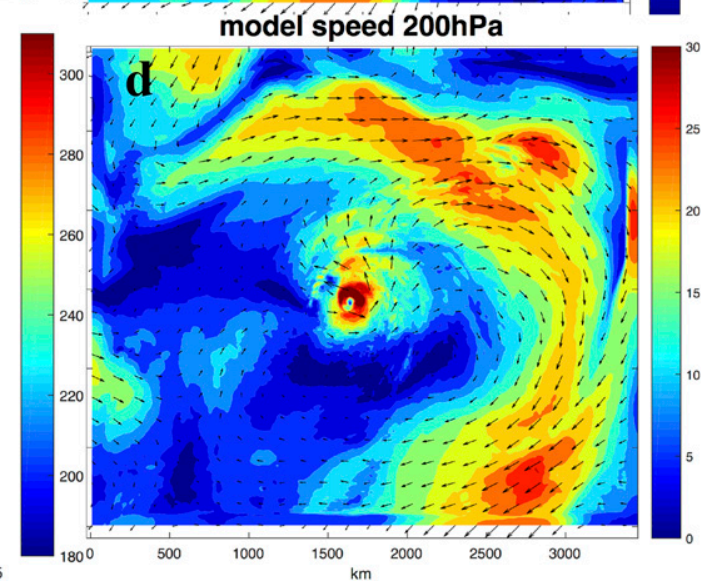

\section{b Reanalysis 200hPa speed}

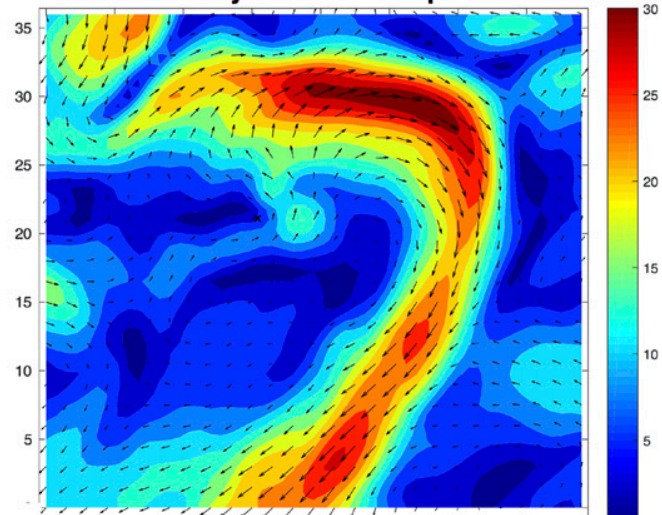



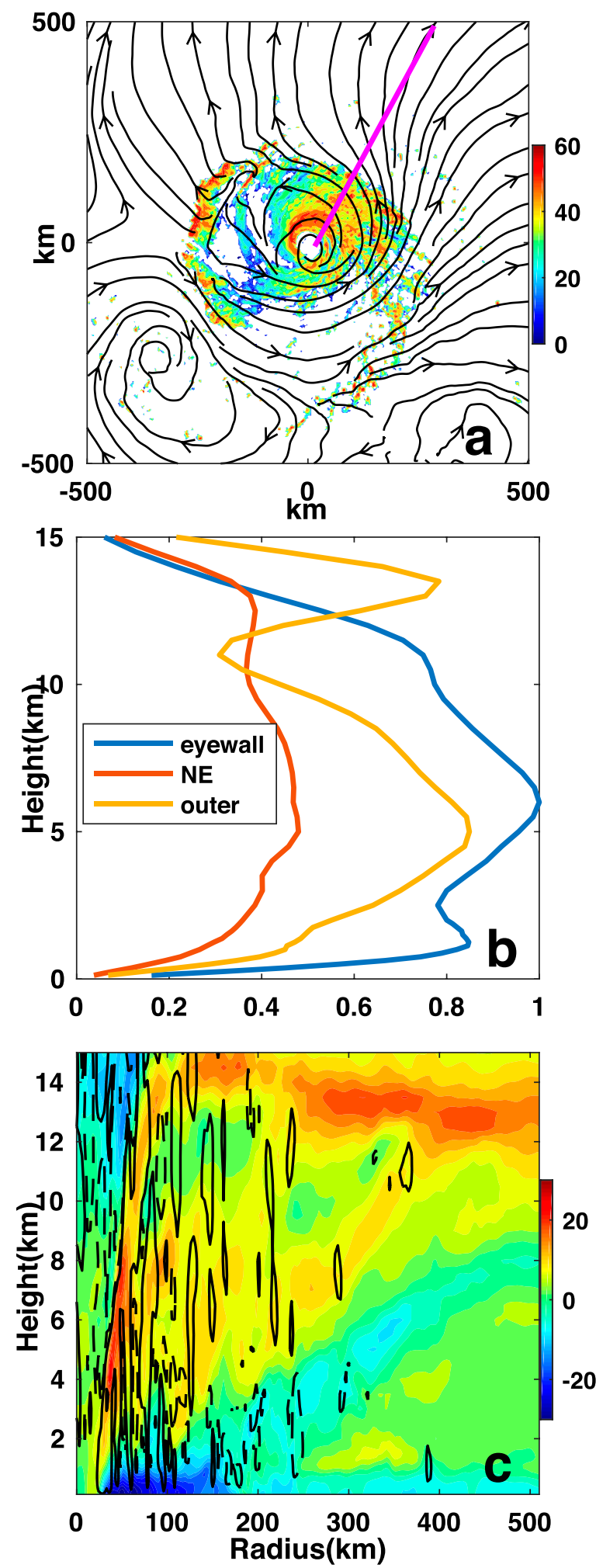

FIG. 13. (a) Simulated reflectivity (shaded) of Hurricane Bill (2009) at 0600 UTC 20 Aug 2009 at $z=2 \mathrm{~km}$ and streamlines (indicating outflow) at $z=12 \mathrm{~km}$. (b) As in Fig. 4, but for the simulation of Bill at 0600 UTC 20 Aug 2009. (c) Vertical cross some experiments, such as $\mathrm{S} 3$, the rainband is weak. This is because in each experiment we have subjectively chosen the model time when the asymmetric rainband just initiated. We find that the stronger the initial TC, the later the outflow-rainband relation develops.

Figure 15 provides a conceptual picture of what is occurring to the TC under the environmental flow: in stage I, a TC is steady under a quiescent environment, with the outflow symmetric and the convection is mostly within the eyewall; in stage II, an upper-level environmental flow (not necessarily a uniform shear flow) approaches the TC, causing one side of the outflow to be enhanced while the other is weakened. At the same time, a descending inflow is induced below the enhanced outflow, and an outer rainband starts to form. In stage III, the enhanced outflow, the descending inflow, and the outer rainband strengthen further under the environmental flow. The rainband also provides the additional source for that enhanced outflow.

\section{Conclusions}

In this paper, we have investigated the relationship between the TC outflow channel induced by the environmental flow and the rainband outside the TC eyewall. By analyzing idealized "JET" simulations of a TC in the presence of an environmental flow, the asymmetric rainband outside the eyewall but within about $200 \mathrm{~km}$ of the center is found to be an important source of the outflow based on the vertical mass flux calculation, even compared against the eyewall. This finding is different from the "NOJET" simulation of a $\mathrm{TC}$ in a quiescent environment, where only the eyewall convection contributes to the upper level. Trajectory analyses show that parcels from the rainband region can go directly to the upper-level outflow. A damped rainband simulation demonstrates that a weakened rainband results in a weakened upper-level radial wind. A realistic numerical simulation of Hurricane Bill (2009) confirms several characteristics of the outflow-rainband relationship as described in the idealized simulations. Additional sensitivity experiments indicate that the outflow-rainband relationship is not very sensitive to variations of the environmental flow or initial TC intensity.

section in the north-northeast direction indicated by the magenta line in (a) of radial wind (shaded) and vertical velocity (solid contours indicate updrafts of 0.5 and $4 \mathrm{~m} \mathrm{~s}^{-1}$; dashed contours indicate downdrafts of -4 and $-0.5 \mathrm{~m} \mathrm{~s}^{-1}$ ). 

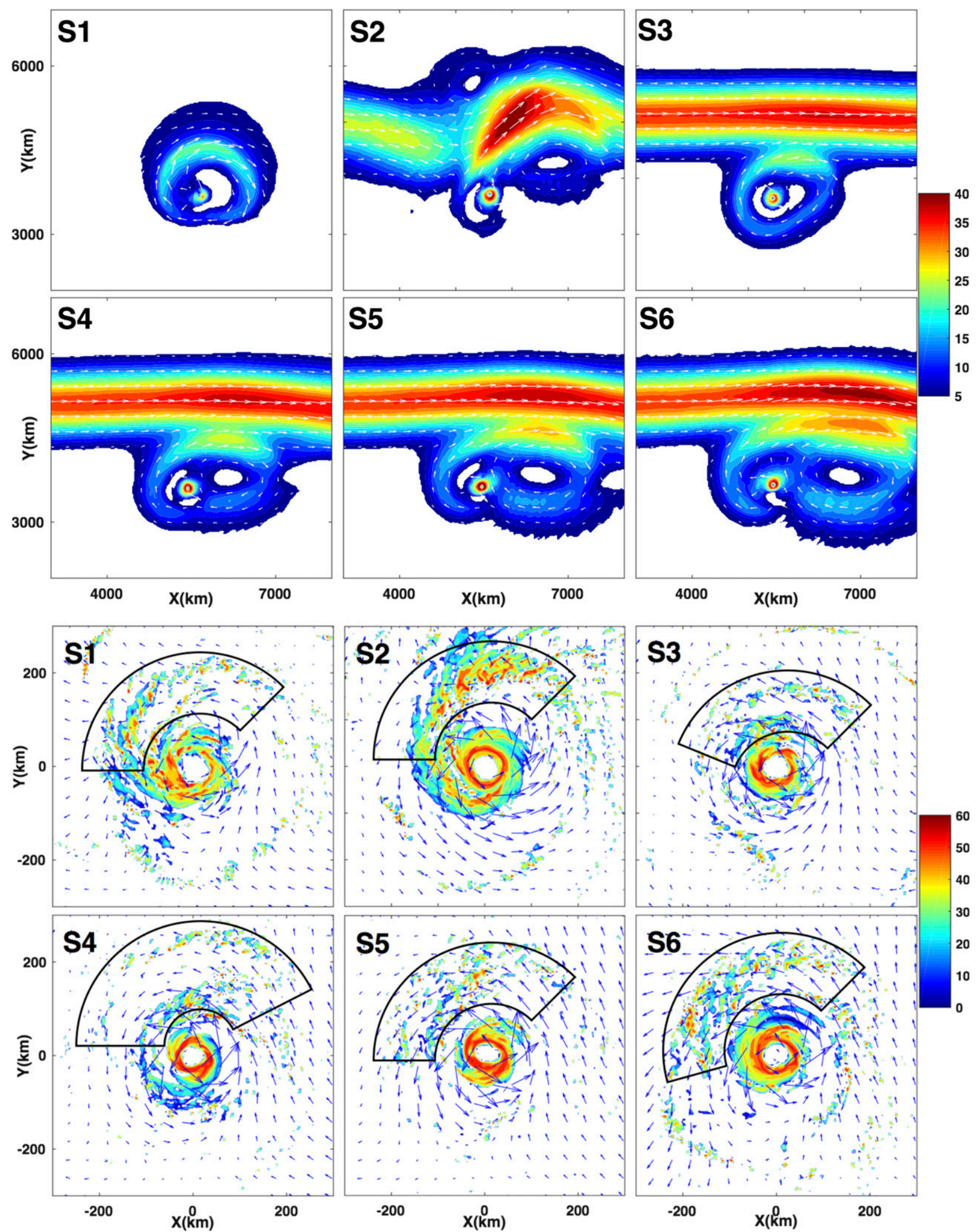

FIG. 14. (top two rows) As in Fig. 2a, but for the upper-level large-scale flow in experiments S1-S6. (bottom two rows) As in Fig. 2e, but for the corresponding inner-core feature in S1-S6. The black-outlined sector indicates asymmetric convection outside the eyewall. The model times for S1-S6 are $t=66,42,36,48,60$, and $66 \mathrm{~h}$, respectively. 


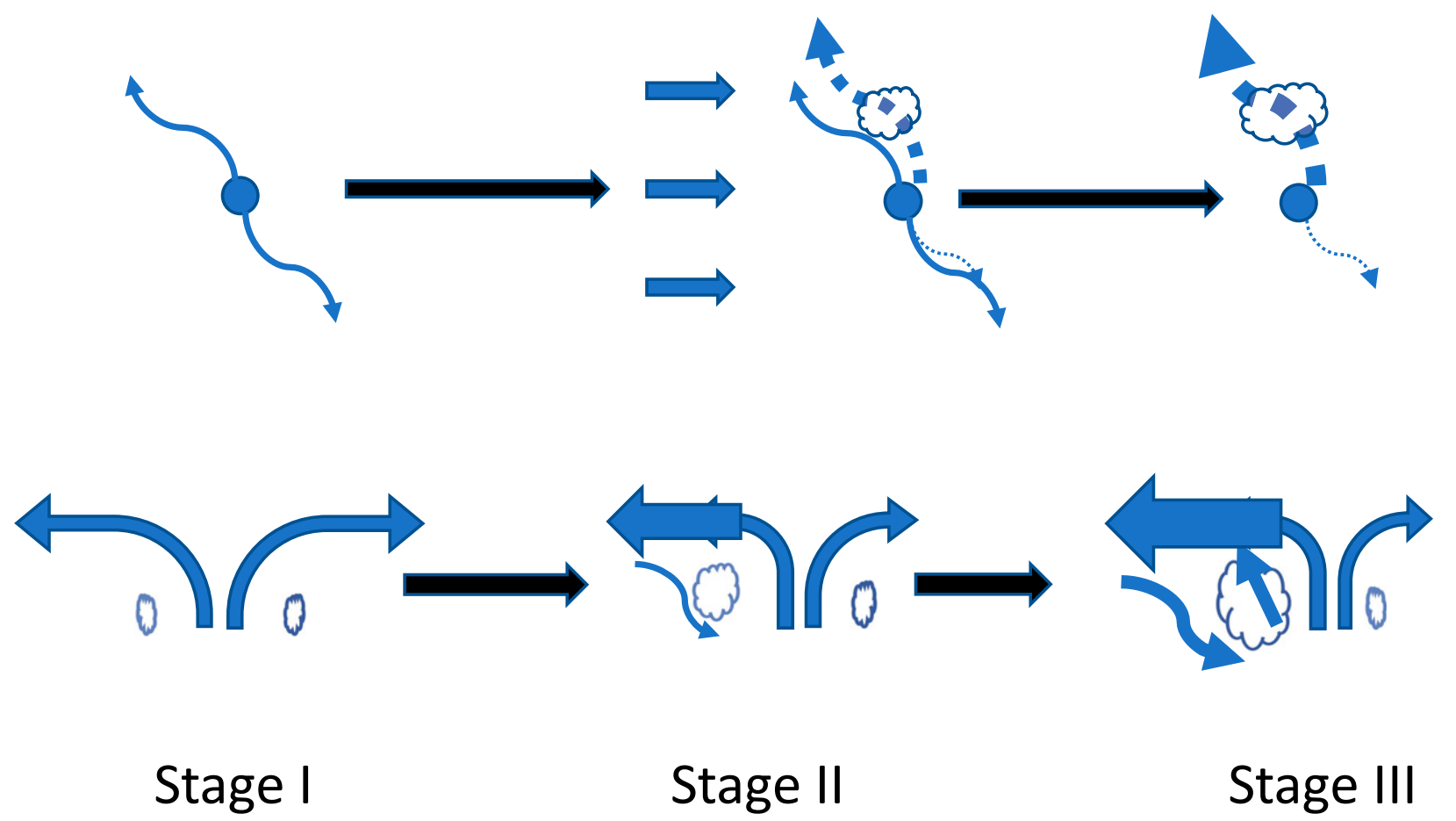

FIG. 15. Conceptual picture of how the outflow and the rainband are affected by the environmental flow. (top) Upper-level horizontal view of the flow and (bottom) vertical cross section of the TC outflow.

We have also sought to understand the mechanism behind the rainband and outflow relationship. Given the fact that the rainband and outflow develop nearly simultaneously, we hypothesize that they are coupled features, both of which belong to the TC response to the environmental flow. The outer rainband and the upperlevel environmental flow is connected by the descending inflow, a persistent feature that originates just below the enhanced outflow at approximately $z=10 \mathrm{~km}$ and descends all way down to the boundary layer near the rainband. This descending inflow, possibly a result of a dynamical response to the enhanced outflow and subsequent diabatic cooling, helps organize the rainband by converging the boundary layer inflow to the rainband region, which in turn provides the air source to the enhanced upper-level outflow. Given stronger environmental flow near the TC inner core, the descending inflow also has a profound impact on TC intensity change (not shown). Therefore, the descending inflow deserves more attention and will be the topic of a future study.

We provided a conceptual model in Fig. 15 to illustrate how the environmental flow creates the asymmetric convection outside the eyewall as well as the outflow channel. Prior to the environmental flow interaction, the outflow is symmetric in the upper troposphere, and convection is mainly concentrated within the eyewall. When an upper-level environmental feature is imposed to the TC, the outflow on one side is enhanced (while the other side is suppressed), because of the low inertial stability in the outflow region. The rainband also starts to form below the outflow. In the final stage, the outflow, outer rainband, and descending inflow all strengthen under the environmental flow.

In nature, the environmental flow around a TC is seldom uniform. One needs to be cautious when using the vertical wind shear to represent the TC environmental flow, because at certain stages the calculated shear may only indicate the information of the relatively large-scale outflow channel, rather than the structure of the environmental flow. The outflow may be a better way to represent the effects of the upper-level environmental flow. While the idealized simulations shown here present a strong relationship between the outflow and TC rainband, more observations and realistic numerical simulations are needed to confirm its presence and variability.

Acknowledgments. This study was supported by the Office of Naval Research Grant N000141410115. The authors also acknowledge helpful discussions with Brian Mapes, Robert Rogers, and Mohamed Iskandarani and computational support from the University of Miami Center for Computational Science. The authors also 
thank two anonymous reviewers for the helpful comments.

\section{REFERENCES}

Bister, M., and K. A. Emanuel, 1997: The genesis of Hurricane Guillermo: TEXMEX analyses and a modeling study. Mon. Wea. Rev., 125, 2662-2682, https://doi.org/10.1175/1520-0493(1997) 125<2662:TGOHGT>2.0.CO;2.

Chen, L. S., and W. M. Gray, 1985: Global view of the upper level outflow pattern associated with tropical cyclone intensity changes during FGGE. Colorado State University Dept. of Atmospheric Science Paper 392, 120 pp.

Chen, S. S., J. A. Knaff, and F. D. Marks, 2006: Effects of vertical wind shear and storm motion on tropical cyclone rainfall asymmetries deduced from TRMM. Mon. Wea. Rev., 134, 3190-3208, https://doi.org/10.1175/MWR3245.1.

Corbosiero, K. L., and J. Molinari, 2003: The relationship between storm motion, vertical wind shear, and convective asymmetries in tropical cyclones. J. Atmos. Sci., 60, 366-376, https:// doi.org/10.1175/1520-0469(2003)060<0366:TRBSMV> 2.0.CO;2.

Dai, Y., S. J. Majumdar, and D. S. Nolan, 2017: Secondary eyewall formation in tropical cyclones by outflow-jet interaction. J. Atmos. Sci., 74, 1941-1958, https://doi.org/10.1175/JAS-D16-0322.1.

Dee, D., and Coauthors, 2011: The ERA-Interim reanalysis: Configuration and performance of the data assimilation system. Quart. J. Roy. Meteor. Soc., 137, 553-597, https://doi.org/ 10.1002/qj.828.

Didlake, A. C., P. D. Reasor, R. F. Rogers, and W. Lee, 2018: Dynamics of the transition from spiral rainbands to a secondary eyewall in Hurricane Earl (2010). J. Atmos. Sci., 75, 2909-2929, https://doi.org/10.1175/JAS-D-17-0348.1.

Ditchek, S. D., J. Molinari, and D. Vollaro, 2017: Tropical cyclone outflow-layer structure and balanced response to eddy forcings. J. Atmos. Sci., 74, 133-149, https://doi.org/10.1175/JASD-16-0117.1.

Draxler, R. R., and G. D. Hess, 1998: An overview of the HYSPLIT_4 modelling system for trajectories, dispersion, and deposition. Aust. Meteor. Mag., 47, 295-308.

Eliassen, A., 1951: Slow thermally or frictionally controlled meridional circulation in a circular vortex. Astrophys. Nor., 5, 19-60.

Emanuel, K. A., 1986: An air-sea interaction theory for tropical cyclones. Part I: Steady-state maintenance. J. Atmos. Sci., 43, 585-605, https://doi.org/10.1175/1520-0469(1986)043<0585: AASITF $>2.0 . \mathrm{CO} ; 2$.

Finocchio, P. M., and S. J. Majumdar, 2017: The predictability of idealized tropical cyclones in environments with time-varying vertical wind shear. J. Adv. Model. Earth Syst., 9, 2836-2862, https://doi.org/10.1002/2017MS001168.

D. S. Nolan, and M. Iskandarani, 2016: Idealized tropical cyclone responses to the height and depth of environmental vertical wind shear. Mon. Wea. Rev., 144, 2155-2175, https://doi.org/10.1175/MWR-D-15-0320.1.

Fischer, M. S., B. H. Tang, and K. L. Corbosiero, 2017: Assessing the influence of upper-tropospheric troughs on tropical cyclone intensification rates after genesis. Mon. Wea. Rev., 145, 12951313, https://doi.org/10.1175/MWR-D-16-0275.1.

Hanley, D., J. Molinari, and D. Keyser, 2001: A composite study of the interaction between tropical cyclones and upper-tropospheric troughs. Mon. Wea. Rev., 129, 2570-2584, https://doi.org/10.1175/ 1520-0493(2001)129<2570:ACSOTI>2.0.CO;2.
Hong, S.-Y., and J.-O. J. Lim, 2006: The WRF single-moment 6-class microphysics scheme (WSM6). J. Korean Meteor. Soc., 42, 129-151.

— J. J. Dudhia, and S.-H. Chen, 2004: A revised approach to ice microphysical processes for the bulk parameterization of clouds and precipitation. Mon. Wea. Rev., 132, 103-120, https://doi.org/10.1175/1520-0493(2004)132<0103:ARATIM> 2.0.CO;2.

_- Y. Noh, and J. Dudhia, 2006: A new vertical diffusion package with an explicit treatment of entrainment processes. Mon. Wea. Rev., 134, 2318-2341, https://doi.org/10.1175/MWR3199.1.

Jones, S. C., 1995: The evolution of vortices in vertical shear: I: Initially barotropic vortices. Quart. J. Roy. Meteor. Soc., 121, 821-851, https://doi.org/10.1002/qj.49712152406.

Klotz, B. W., and D. S. Nolan, 2019: SFMR surface wind undersampling over the tropical cyclone life cycle. Mon. Wea. Rev., 147, 247-268, https://doi.org/10.1175/MWR-D-18-0296.1.

Knapp, K. R., and Coauthors, 2011: Globally gridded satellite observations for climate studies. Bull. Amer. Meteor. Soc., 92, 893-907, https://doi.org/10.1175/2011BAMS3039.1.

Molinari, J., and D. Vollaro, 1989: External influences on hurricane intensity. Part I: Outflow layer eddy angular momentum fluxes. J. Atmos. Sci., 46, 1093-1105, https://doi.org/10.1175/ 1520-0469(1989)046<1093:EIOHIP>2.0.CO;2.

— Part II: Vertical structure and response of the hurricane vortex. J. Atmos. Sci., 47, 1902-1918, https://doi.org/10.1175/15200469(1990)047<1902:EIOHIP>2.0.CO;2.

$\longrightarrow$, and _ 2014: Symmetric instability in the outflow layer of a major hurricane. J. Atmos. Sci., 71, 3739-3746, https://doi.org/ 10.1175/JAS-D-14-0117.1.

Moon, Y., and D. S. Nolan, 2015: Spiral rainbands in a numerical simulation of Hurricane Bill (2009). Part I: Structures and comparisons to observations. J. Atmos. Sci., 72, 164-190, https://doi.org/10.1175/JAS-D-14-0058.1.

Nolan, D. S., 2011: Evaluating environmental favorableness for tropical cyclone development with the method of point downscaling. J. Adv. Model. Earth Syst., 3, M08001, https:// doi.org/10.1029/2011MS000063.

_ R. R. Atlas, K. T. Bhatia, and L. R. Bucci, 2013: Development and validation of a hurricane nature run using the joint OSSE nature run and the WRF Model. J. Adv. Model. Earth Syst., 5, 382-405, https://doi.org/10.1002/jame.20031.

Onderlinde, M. J., and D. S. Nolan, 2017: The tropical cyclone response to changing wind shear using the method of timevarying point-downscaling. J. Adv. Model. Earth Syst., 9, 908-931, https://doi.org/10.1002/2016MS000796.

Ooyama, K. V., 1987: Numerical experiments of steady and transient jets with simple model of the hurricane outflow layer. Preprints, 17th Conf. on Hurricanes and Tropical Meteorology, Miami, FL, Amer. Meteor. Soc., 318-320.

Peirano, C. M., K. L. Corbosiero, and B. H. Tang, 2016: Revisiting trough interactions and tropical cyclone intensity change. Geophys. Res. Lett., 43, 5509-5515, https://doi.org/10.1002/ 2016 GL069040.

Pfeffer, R. L., and M. Challa, 1981: A numerical study of the role of eddy fluxes of momentum in the development of Atlantic hurricanes. J. Atmos. Sci., 38, 2393-2398, https://doi.org/ 10.1175/1520-0469(1981)038<2393:ANSOTR >2.0.CO;2.

Reasor, P. D., M. T. Montgomery, and L. D. Grasso, 2004: A new look at the problem of tropical cyclones in vertical shear flow: Vortex resiliency. J. Atmos. Sci., 61, 3-22, https://doi.org/ 10.1175/1520-0469(2004)061<0003:ANLATP>2.0.CO;2. 
Riemer, M., M. T. Montgomery, and M. E. Nicholls, 2010: A new paradigm for intensity modification of tropical cyclones: Thermodynamic impact of vertical wind shear on the inflow layer. Atmos. Chem. Phys., 10, 3163-3188, https://doi.org/10.5194/acp-10-3163-2010.

Rogers, R., S. S. Chen, J. Tenerelli, and H. Willoughby, 2003: A numerical study of the impact of vertical shear on the distribution of rainfall in Hurricane Bonnie (1998). Mon. Wea. Rev., 131, 1577-1599, https://doi.org/10.1175//2546.1.

_ J. J. Zhang, J. Zawislak, H. Jiang, G. R. Alvey III, E. J. Zipser, and S. N. Stevenson, 2016: Observations of the structure and evolution of Hurricane Edouard (2014) during intensity change. Part II: Kinematic structure and the distribution of deep convection. Mon. Wea. Rev., 144, 3355-3376, https://doi.org/10.1175/ MWR-D-16-0017.1.

Romps, D. M., and Z. Kuang, 2010: Do undiluted convective plumes exist in the upper tropical troposphere? J. Atmos. Sci., 67, 468-484, https://doi.org/10.1175/2009JAS3184.1.

Shapiro, L. J., and M. T. Montgomery, 1993: A three-dimensional balance theory for rapidly rotating vortices. J. Atmos. Sci., $\mathbf{5 0}$, 3322-3335, https://doi.org/10.1175/1520-0469(1993)050<3322: ATDBTF $>2.0 . \mathrm{CO} ; 2$.
Shi, J.-J., S. W.-J. Chang, and S. Raman, 1990: A numerical study of the outflow layer of tropical cyclones. Mon. Wea. Rev., 118, 2042-2055, https://doi.org/10.1175/1520-0493(1990)118<2042: ANSOTO $>2.0 . \mathrm{CO} ; 2$.

Simmons, A. J., and B. J. Hoskins, 1977: Baroclinic instability on the sphere: Solutions with a more realistic tropopause. J. Atmos. Sci., 34, 581-588, https://doi.org/10.1175/1520-0469(1977) $034<0581$ :BIOTSS $>2.0$.CO 2 .

Stevens, D. E., and P. E. Ciesielski, 1986: Inertial instability of horizontally sheared flow away from the equator. J. Atmos. Sci., 43, 2845-2856, https://doi.org/10.1175/1520-0469(1986) 043<2845:IIOHSF $>2.0$.CO;2.

Velden, C. S., C. M. Hayden, S. J. Nieman, W. P. Menzel, S. Wanzong, and J. S. Goerss, 1997: Upper-tropospheric winds derived from geostationary satellite water vapor observations. Bull. Amer. Meteor. Soc., 78, 173-196, https:// doi.org/10.1175/1520-0477(1997)078<0173:UTWDFG> 2.0.CO;2.

Wang, Y., 2009: How do outer spiral rainbands affect tropical cyclone structure and intensity? J. Atmos. Sci., 66, 1250-1273, https://doi.org/10.1175/2008JAS2737.1. 